\title{
How to access QED at a supercritical Coulomb field
}

\author{
R. V. Popov $\odot,{ }^{1}$ V. M. Shabaev $\odot,{ }^{1, *}$ D. A. Telnov $\odot,{ }^{1}$ I. I. Tupitsyn,,${ }^{1}$ I. A. Maltsev $\odot,{ }^{1}$ Y. S. Kozhedub $\odot,{ }^{1}$ \\ A. I. Bondarev $\odot{ }^{2}$ N. V. Kozin $\odot,{ }^{1}$ X. Ma, ${ }^{3}$ G. Plunien $\odot,{ }^{4}$ T. Stöhlker, ${ }^{5,6,7}$ D. A. Tumakov $\oplus^{1}$, and V. A. Zaytsev $\circledast^{1}$ \\ ${ }^{1}$ Department of Physics, St. Petersburg State University, \\ Universitetskaya 7/9, 199034 St. Petersburg, Russia \\ ${ }^{2}$ Center for Advanced Studies, Peter the Great St. Petersburg Polytechnic University, Polytekhnicheskaja \\ 29, 195251 St. Petersburg, Russia \\ ${ }^{3}$ Institute of Modern Physics, Chinese Academy of Sciences, Nanchang Road 509, 730000 Lanzhou, China \\ ${ }^{4}$ Institut für Theoretische Physik, TU Dresden, D-01062 Dresden, Germany \\ ${ }^{5}$ GSI Helmholtzzentrum für Schwerionenforschung GmbH, D-64291 Darmstadt, Germany \\ ${ }^{6}$ Helmholtz-Institut Jena, D-07743 Jena, Germany \\ ${ }^{7}$ Institut für Optik und Quantenelektronik, Friedrich-Schiller-Universität Jena, D-07743 Jena, Germany
}

(Received 11 August 2020; accepted 23 September 2020; published 13 October 2020)

In slow collisions of two bare nuclei with the total charge number larger than the critical value, $Z_{\mathrm{cr}} \approx 173$, the initially neutral vacuum can spontaneously decay into the charged vacuum and two positrons. Detection of the spontaneous emission of positrons would be the direct evidence of this fundamental phenomenon. However, the spontaneous emission is generally masked by the dynamical positron emission, which is induced by a strong time-dependent electric field created by the colliding nuclei. In our recent paper [I. A. Maltsev et al., Phys. Rev. Lett. 123, 113401 (2019)] it has been shown that the spontaneous pair production can be observed via measurements of the pair-production probabilities for a given set of nuclear trajectories. In the present paper, we have significantly advanced this study by exploring additional aspects of the process we are interested in. We calculate the positron energy spectra and find that these spectra can give a clear signature of the transition from the subcritical to the supercritical regime. It is found that focusing on a part of the positron spectrum, which accounts for the energy region where the spontaneously created positrons can contribute, allows us to get a much stronger evidence of the transition to the supercritical mode, making it very well pronounced in collisions, for example, of two uranium nuclei. The possibility of extending this study to collisions of bare nuclei with neutral atoms is also considered. The probability of a vacancy in the lowest-energy state of a quasimolecule which is formed in collisions of a bare $\mathrm{U}$ nucleus with neutral $\mathrm{U}$ and $\mathrm{Cm}$ atoms has been calculated. The relatively large values of this probability make such collisions suitable for observing the vacuum decay.

DOI: 10.1103/PhysRevD.102.076005

\section{INTRODUCTION}

After the foundations of quantum field theory were formulated in the early 1930s, it was shown that the theory predicts the spontaneous creation of electron-positron pairs by a constant uniform electric field if the strength of the field is comparable to or greater than a critical value, $E_{\mathrm{cr}}=m_{e}^{2} c^{3} /(|e| \hbar) \approx 1.3 \times 10^{16} \mathrm{~V} / \mathrm{cm}$, [1-3]. While from the point of view of the theory this phenomenon was studied in a large number of works (see Refs. [4-16] and references therein), its experimental detection was not

\footnotetext{
*Corresponding author: v.shabaev@spbu.ru
}

Published by the American Physical Society under the terms of the Creative Commons Attribution 4.0 International license. Further distribution of this work must maintain attribution to the author(s) and the published article's title, journal citation, and DOI. Funded by SCOAP. possible because of practical inaccessibility of the required field strength. Some hopes for experimental detection of this fundamental effect were associated with the development of novel laser technologies [17,18]. But, apparently, in the foreseeable future, it is quite unlikely to realize a possible experiment in the required strong field regime solely on the basis of high-power laser.

An alternative approach to the study of vacuum properties in the presence of a supercritical field was proposed in the works of Soviet and German physicists [19-34]. It is known that in the case of a pure Coulomb field induced by a pointlike charge $Z$ the 1 s level exists only up to $Z \approx 137$ and then disappears. However, for extended nuclei this level goes continuously down and at $Z=Z_{\mathrm{cr}} \approx 173$ reaches the onset of the negative-energy Dirac continuum (see Fig. 1). If this level was empty, it dives into the negativeenergy continuum as a resonance, the decay of which should lead to a spontaneous creation of positrons. Namely, 


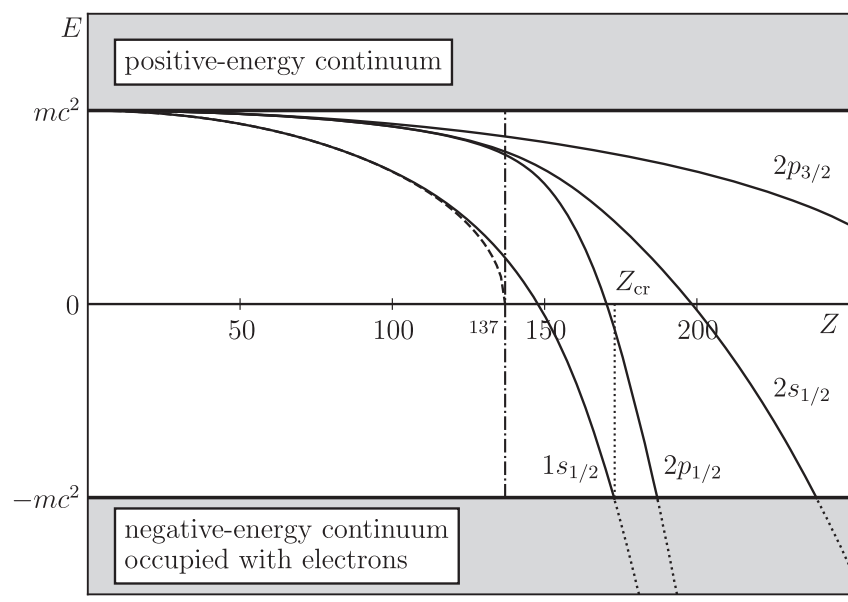

FIG. 1. The low-lying energy levels of a H-like ion as functions of the nuclear charge number $Z$.

when switching from the subcritical to the supercritical mode, the initially neutral vacuum decays into a charged vacuum and two positrons (due to spin degeneracy). Since there are no nuclei with such a large charge in nature (the charge of the heaviest element discovered to date, oganesson, is 118), the only way to create a supercritical Coulomb field is to collide nuclei with the total charge number greater than the critical value $\left(Z_{1}+Z_{2}>173\right)$. The time dependence of the quasimolecular energy levels in collision of two uranium ions (nuclei) is presented in Fig. 2. Following Greiner and co-authors [32], in this figure various pair-creation mechanisms are conventionally shown by arrows. The arrows $a, b$, and $c$ denote dynamical pair-creation processes and the arrow $d$ indicates the spontaneous pair creation. The dynamical pair creation

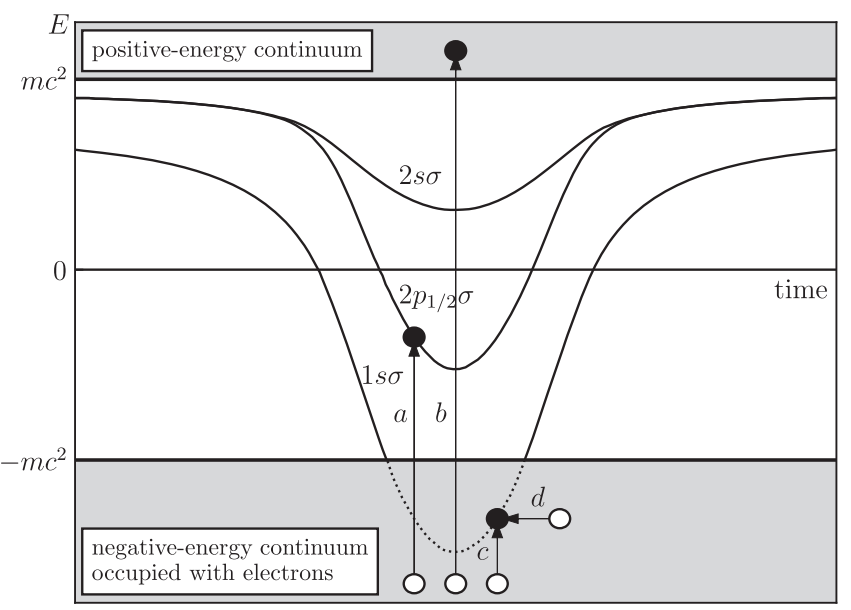

FIG. 2. The low-lying energy levels of a quasimolecule formed in collision of two uranium ions (nuclei) as functions of time. The arrows $a, b$, and $c$ denote different dynamical pair-creation mechanisms and the arrow $d$ indicates the spontaneous pair creation. The $1 s$ state dives into the negative-energy continuum for about $10^{-21} \mathrm{~s}$. takes place in both subcritical and supercritical modes while the spontaneous pair creation starts to work only in the supercritical regime. The dynamical mechanisms $(a, b, c)$ superpose with the spontaneous one $(d)$ and generally cannot be separated from each other. In addition, the spontaneous pair creation is strongly suppressed due to very small period of the supercritical regime time $\left(\sim 10^{-21} \mathrm{~s}\right)$, which is by about two orders of magnitude smaller than the time required for the vacuum decay. All this was one of the main reasons why attempts to experimentally observe the spontaneous creation of positrons, which were undertaken more than 30 years ago in Darmstadt (see, e.g., Ref. [32] and references therein), were not successful. Moreover, the Frankfurt group, which worked on the theory of this phenomenon for more than 20 years, concluded that the vacuum decay can only be observed experimentally if colliding nuclei stick together for some time due to nuclear forces [35,36]. However, no evidence of the nuclear sticking in the collisions of interest has been found to date, making this scenario not promising for future experiments.

Despite the aforementioned conclusions by the Frankfurt group, one could expect, however, that the detailed study of quantum dynamics of the electron-positron field in lowenergy heavy-ion collisions would allow to find some signatures which indicate the principal difference between the subcritical and the supercritical regimes. To carry out these studies, first of all it was necessary to develop the theoretical and computational methods beyond the approximation made by the Frankfurt group. To this end, more than a decade ago new efforts have been initiated by the St. Petersburg group [37-44]. These methods allowed the calculations of the charge-transfer, electron-excitation, ionization, and pair-production processes. In particular, in Refs. [42-44] the first calculations of the pair-production probabilities beyond the monopole approximation, which was widely used by the Frankfurt group, were performed. These calculations showed that effects beyond the monopole approximation only slightly change the pair-creation probabilities in the region of small impact parameters. This has provided much more flexibility in studying various scenarios of finding the signatures which can demonstrate the principal difference between the subcritical and the supercritical regimes. As a result of these studies, in Ref. [45] it was found that the vacuum decay can be observed via impact-sensitive measurements of pair-production probabilities. In this paper, we present additional aspects of this study and find important signatures of the principal difference between the subcritical and supercritical regimes, which can be observed in the positron spectra.

In the next sections we will mainly consider quantum dynamics of electron-positron field in collisions of heavy bare nuclei. Experiments on low-energy collisions of bare nuclei will be possible at the future facilities in Germany (GSI/FAIR) [46-48], China (HIAF) [49], and Russia 
(NICA) [50]. After the formulation of the basic theory, we will present the results of the calculations of the paircreation probabilities and positron spectra as functions of the nuclear charges and the collision energy for the trajectories with a given minimal internuclear distance. The dependence of the pair-creation probabilities and the positron spectra on the value of the minimal internuclear distance will be also studied. It will be shown that the study of a partial probability, which accounts for the region of the positron energy distribution where the spontaneously created positrons can contribute, allows a significant increase of the signature of the transition to the supercritical regime, compared to the analysis of the total probability. At the end of the paper we will discuss a possibility for studying the proposed scenarios on the vacuum decay observation in collisions of bare nuclei with neutral atoms.

The relativistic units $(\hbar=c=1)$ and the Heaviside charge unit $\left(\alpha=e^{2} /(4 \pi), e<0\right)$ are used throughout the paper.

\section{THEORY}

\section{A. General formalism}

To study the quantum dynamics of the electron-positron field in low-energy collisions of heavy nuclei, one can use the approach, where the relative motion of the colliding nuclei is treated classically [24,32]. In this approach, to derive the pair-creation probabilities, one has to consider first the solutions of the Dirac equation in the external timedependent potential induced by the colliding nuclei, whose motion is described by the Rutherford trajectories. For the relatively slow collisions of interest the magnetic part of the potential can be neglected and the time-dependent Dirac equation (TDDE) takes the form:

$$
i \frac{\partial}{\partial t} \psi(\boldsymbol{r}, t)=H(t) \psi(\boldsymbol{r}, t)
$$

with

$$
H(t)=\boldsymbol{\alpha} \cdot \boldsymbol{p}+\beta m_{e}+V(\boldsymbol{r}, t) .
$$

Here $\boldsymbol{\alpha}, \beta$ are the Dirac matrices, $m_{e}$ is the electron mass, and $V(\boldsymbol{r}, t)$ is the total two-center potential induced by the nuclei:

$$
V(\boldsymbol{r}, t)=V_{\mathrm{A}}\left(\left|\boldsymbol{r}-\boldsymbol{R}_{\mathrm{A}}(t)\right|\right)+V_{\mathrm{B}}\left(\left|\boldsymbol{r}-\boldsymbol{R}_{\mathrm{B}}(t)\right|\right),
$$

where the vectors $\boldsymbol{R}_{\mathrm{A}}$ and $\boldsymbol{R}_{\mathrm{B}}$ denote the positions of the colliding nuclei and

$$
V_{\mathrm{A}, \mathrm{B}}(\boldsymbol{r})=\frac{e}{4 \pi} \int d \boldsymbol{r}^{\prime} \frac{\rho_{\mathrm{A}, \mathrm{B}}\left(\boldsymbol{r}^{\prime}\right)}{\left|\boldsymbol{r}-\boldsymbol{r}^{\prime}\right|}
$$

are the corresponding nuclear potentials. For the nuclear charge distribution $\rho(\boldsymbol{r})$ we utilize the model of the uniformly charged sphere.
Considering the quantum dynamics from the initial time $t_{\text {in }}$ to the final time $t_{\text {out }}$, one can define two sets of solutions of the TDDE, which have the asymptotics:

$$
\psi_{i}^{(+)}\left(\boldsymbol{r}, t_{\text {in }}\right)=\phi_{i}^{\text {in }}(\boldsymbol{r}), \quad \psi_{i}^{(-)}\left(\boldsymbol{r}, t_{\text {out }}\right)=\phi_{i}^{\text {out }}(\boldsymbol{r}),
$$

where $\phi_{i}^{\text {in }}(\boldsymbol{r})$ and $\phi_{i}^{\text {out }}(\boldsymbol{r})$ are the eigenfunctions of the Dirac Hamiltonian at the corresponding time moments,

$$
\begin{gathered}
H\left(t_{\text {in }}\right) \phi_{i}^{\text {in }}(\boldsymbol{r})=\varepsilon_{i}^{\text {in }} \phi_{i}^{\text {in }}(\boldsymbol{r}), \\
H\left(t_{\text {out }}\right) \phi_{i}^{\text {out }}(\boldsymbol{r})=\varepsilon_{i}^{\text {out }} \phi_{i}^{\text {out }}(\boldsymbol{r}) .
\end{gathered}
$$

The most natural way to derive formulas for the paircreation probabilities is to use the second quantization formalism [4]. To this end, we introduce the "in" and "out" vacuum states, $\mid 0$, in $\rangle$ and $\mid 0$, out $\rangle$, and the related annihilation operators:

$$
\left.\left.\hat{b}_{i}^{\text {(in) }} \mid 0, \text { in }\right\rangle=0, \quad \hat{b}_{i}^{\text {(out) })} \mid 0, \text { out }\right\rangle=0
$$

for particles, which we will denote symbolically by " $i>F$ ", where $F$ stands for the "Fermi level," and

$$
\left.\left.\hat{d}_{i}^{\text {(in) }} \mid 0, \text { in }\right\rangle=0, \quad \hat{d}_{i}^{\text {(out) })} \mid 0, \text { out }\right\rangle=0
$$

for antiparticles (" $i<F$ "). These operators obey the standard anticommutation relations. The electron-positron field operator $\hat{\Psi}(\boldsymbol{r}, t)$ in the Heisenberg picture is defined as

$$
\begin{array}{r}
\hat{\Psi}(\boldsymbol{r}, t)=\sum_{i>F} \hat{b}_{i}^{(\mathrm{in})} \psi_{i}^{(+)}(\boldsymbol{r}, t)+\sum_{i<F} \hat{d}_{i}^{(\mathrm{in}) \dagger} \psi_{i}^{(+)}(\boldsymbol{r}, t), \\
\hat{\Psi}(\boldsymbol{r}, t)=\sum_{i>F} \hat{b}_{i}^{(\text {out })} \psi_{i}^{(-)}(\boldsymbol{r}, t)+\sum_{i<F} \hat{d}_{i}^{(\text {out }) \dagger} \psi_{i}^{(-)}(\boldsymbol{r}, t) .
\end{array}
$$

For collisions of bare nuclei, the initial $t=t_{\text {in }}$ state of the system is described by the vacuum vector $\mid 0$, in $\rangle$. To find the number of the electrons in a state " $k$ " at $t=t_{\text {out }}$, one should evaluate the value $n_{k}=\left\langle 0\right.$, in $\left|\hat{b}_{k}^{\text {(out }) \dagger} \hat{b}_{k}^{\text {(out) }}\right| 0$, in $\rangle$. This can be done by employing the Eqs. (10)-(11) and the anticommutation relations between the creation and annihilation operators. As the result, one obtains $[4,32]$

$$
n_{k}=\left\langle 0, \text { in }\left|\hat{b}_{k}^{\text {(out } \dagger \dagger} \hat{b}_{k}^{(\text {out })}\right| 0, \text { in }\right\rangle=\sum_{i<F}\left|a_{k i}\right|^{2},
$$

where

$$
a_{i j}=\int d \boldsymbol{r} \psi_{i}^{(-) \dagger}(\boldsymbol{r}, t) \psi_{j}^{(+)}(\boldsymbol{r}, t)
$$

is the one-electron transition amplitude. Since the amplitudes $a_{i j}$ are time-independent, they can be evaluated at the time moment $t_{\text {in }}$ or $t_{\text {out }}$ : 


$$
\begin{aligned}
a_{i j} & =\int d \boldsymbol{r} \psi_{i}^{(-) \dagger}\left(\boldsymbol{r}, t_{\text {in }}\right) \phi_{j}^{\text {in }}(\boldsymbol{r}) \\
& =\int d \boldsymbol{r} \phi_{i}^{\text {out } \dagger}(\boldsymbol{r}) \psi_{j}^{(+)}\left(\boldsymbol{r}, t_{\text {out }}\right) .
\end{aligned}
$$

Alternatively, one can calculate the number of created positrons in a state " $p$ " by formula

$$
\bar{n}_{p}=\left\langle 0, \text { in }\left|\hat{d}_{p}^{(\text {out }) \dagger} \hat{d}_{p}^{\text {(out) }}\right| 0, \text { in }\right\rangle=\sum_{i>F}\left|a_{p i}\right|^{2} .
$$

The total number of the electron-positron pairs can be found as

$$
P=\sum_{k>F} n_{k}=\sum_{p<F} \bar{n}_{p}
$$

Taking into account that for the processes under consideration $P \ll 1$, we will refer to this value as the paircreation probability.

For the calculations of $n_{k}$ and $\bar{n}_{p}$ it is convenient to use the finite basis set method $[39,40,42-44,51,52]$. With this method, one gets a spectrum consisting of a finite number of states, including both bound and continuum (positiveand negative-energy) pseudostates. As a result, all the related summations in the equations given above run over a finite number of states.

Finally, it should be emphasized that the formulas presented in this section account for all pair-creation channels $(a, b, c$, and $d)$ shown in Fig. 2.

\section{B. Monopole approximation}

Numerical solution of the TDDE with the total twocenter potential (3) is very time consuming. For this reason, the calculations of the pair-creation probabilities are generally restricted to the so-called monopole approximation for $V(\boldsymbol{r}, t)$. In this approximation, the potential $V(\boldsymbol{r}, t)$ is expanded in spherical harmonics in the center-of-mass frame and only the lowest-order spherical-symmetric term of this expansion is taken into account,

$$
V^{(\text {mon })}(r, t)=\frac{1}{4 \pi} \int d \boldsymbol{n} V(\boldsymbol{r}, t),
$$

where $\boldsymbol{n}=\boldsymbol{r} / r$. The direct calculations of the pair-creation probabilities and the positron spectra near their maxima for the two-center potential [42-44] have demonstrated that the monopole approximation works rather well, unless the impact parameter of the nuclear collisions is too large. In particular, in the case of uranium-uranium collisions at the projectile energy $6.2 \mathrm{MeV} / \mathrm{u}$ (in the rest frame of the target nucleus before the collision) the difference between the two-center and the monopole-approximation results for the total probability varies from about $6 \%$ for the head-on collision $(b=0)$ to about $10 \%$ at $b=10 \mathrm{fm}$. Since the impact parameter $b=10 \mathrm{fm}$ corresponds to the scattering angle $\theta=79^{\circ}$ in the center-of-mass frame (at the energy under consideration), this means the validity of the monopole approximation is preserved in a wide range of the scattering angles around the backward direction.

For the spherically symmetric potential (17), the Dirac wave functions are represented in the standard form

$$
\psi_{\kappa m}(\boldsymbol{r}, t)=\frac{1}{r}\left(\begin{array}{c}
G_{\kappa}(r, t) \Omega_{\kappa m}(\boldsymbol{n}) \\
i F_{\kappa}(r, t) \Omega_{-\kappa m}(\boldsymbol{n})
\end{array}\right),
$$

where $\Omega_{\kappa m}(\boldsymbol{n})$ is the spherical spinor, $G_{\kappa}(r, t) / r$ and $F_{\kappa}(r, t) / r$ are the radial Dirac components, and $\kappa=$ $(-1)^{j+l+1 / 2}(j+1 / 2)$ is the relativistic quantum number defined by the angular momentum and parity. Due to the conservation of the total angular momentum, one has to deal with the time-dependent radial Dirac equation for a given value of $\kappa$,

$$
i \frac{\partial}{\partial t} \phi(r, t)=H_{\kappa}(t) \phi(r, t)
$$

where

$$
\phi(r, t)=\left(\begin{array}{l}
G(r, t) \\
F(r, t)
\end{array}\right)
$$

is the radial Dirac wave function and

$H_{\kappa}(t)=\left(\begin{array}{cc}m_{e}+V^{(\mathrm{mon})}(r, t) & -\frac{d}{d r}+\frac{\kappa}{r} \\ \frac{d}{d r}+\frac{\kappa}{r} & -m_{e}+V^{(\mathrm{mon})}(r, t)\end{array}\right)$

is the radial Dirac Hamiltonian.

For a given $\kappa$, the initial states, including the bound and continuum pseudostates, are obtained by diagonalization of the matrix $\mathcal{H} \equiv H_{\kappa}\left(t_{\text {in }}\right)$ in a finite basis set. The basis functions are constructed from B-splines [53] within the framework of the dual-kinetic-balance approach [54]. To solve Eq. (19), we expand $\phi(r, t)$ on a basis of the eigenstates of the matrix $\mathcal{H}$. For a given initial condition defined by Eq. (5), we have

$$
\phi_{i}(r, t)=\sum_{k=1}^{N} c_{k i}(t) u_{k}(r) e^{-i \varepsilon_{k} t}
$$

where $N$ is the number of the states, $\varepsilon_{k}$ are the eigenvalues of the $\mathcal{H}$ matrix, and $c_{k i}$ are the expansion coefficients. Substitution of the expansion (22) into Eq. (19) leads to the equations:

$i \frac{\partial}{\partial t} c_{j i}(t)=\sum_{k} V_{j k}(t) c_{k i}(t), \quad$ subject to $c_{j i}\left(t_{\text {in }}\right)=\delta_{j i}$, 
where

$V_{j k}(t)=\left\langle u_{j}\left|\left(V^{(\mathrm{mon})}(r, t)-V^{(\mathrm{mon})}\left(r, t_{\mathrm{in}}\right)\right)\right| u_{k}\right\rangle e^{-i\left(\varepsilon_{k}-\varepsilon_{j}\right) t}$.

The equations (23) are solved using the Crank-Nicolson scheme [55]:

$$
\vec{c}_{i}(t+\Delta t) \approx M(t+\Delta t ; t) \vec{c}_{i}(t),
$$

where $\Delta t$ is a small time step, $\vec{c}_{i}=\left\{c_{1 i}, \ldots, c_{N i}\right\}$, and the $M$ matrix is determined by

$$
\begin{aligned}
M(t+\Delta t ; t)= & {\left[I+i \frac{\Delta t}{2} V\left(t+\frac{\Delta t}{2}\right)\right]^{-1} } \\
& \times\left[I-i \frac{\Delta t}{2} V\left(t+\frac{\Delta t}{2}\right)\right] .
\end{aligned}
$$

With this technique we propagate all the bound and continuum quasistates from $t_{\text {in }}$ to $t_{\text {out }}$ and calculate the pair-creation probability for a given $\kappa$ using the formula (16). The total pair-creation probability is obtained as a sum of the partial $\kappa$ contributions. Usually it is sufficient to account for the $\kappa= \pm 1$ contributions only. This is due to the dominant contribution of the pair-creation processes in which the created electrons are captured into bound states $[33,43,51]$.

The calculation of the positron spectrum can be performed according to Eq. (15). However, due to the use of the finite basis set methods, the direct calculation of the energy-differential spectrum $d P / d \varepsilon$ by this formula is not possible. Therefore, to obtain $d P / d \varepsilon_{p}$ we use the Stieltjes method $[51,52,56]$ :

$$
\frac{d P}{d \varepsilon}\left(\frac{\varepsilon_{p}+\varepsilon_{p+1}}{2}\right)=\frac{1}{2} \frac{\bar{n}_{p+1}+\bar{n}_{p}}{\varepsilon_{p+1}-\varepsilon_{p}},
$$

where $\varepsilon_{p}$ is the eigenvalue of the Hamiltonian in the finite basis set.

\section{Choice of trajectories}

In what follows, we will mainly consider the collisions of bare nuclei at the energies close to the Coulomb barrier, which is defined as the collision energy at which the nuclei touch each other. The nuclear trajectories are defined by nonrelativisic classical mechanics and, in the case of bare nuclei, are given by the well-known equations [57]. According to these equations, the minimal distance between the nuclei, $R_{\min }$, is related to the impact parameter $b$ by

$$
b^{2}=R_{\min }^{2}-\frac{\alpha Z_{1} Z_{2}}{E} R_{\min },
$$

where $E$ is the collision energy in the center-of-mass frame. For a given value of $R_{\min }$, the minimal energy, $E_{0}$, corresponds to the head-on $(b=0)$ collision,

$$
E_{0}=\frac{\alpha Z_{1} Z_{2}}{R_{\min }}
$$

Let us consider only the trajectories which correspond to the same minimal distance $\left(R_{\min }\right)$ for different impact parameters $(b)$ and, therefore, for different collision energies $(E)$. In case of uranium-uranium collisions with $R_{\min }=17.5 \mathrm{fm}$, which corresponds to the projectile energy $E_{0}=5.9 \mathrm{MeV} / \mathrm{u}$ (in the rest frame of the target nucleus before the collision), the trajectories of interest are shown in Fig. 3 in the center-of-mass frame of reference. In addition to the circle, corresponding to $R_{\min }=17.5 \mathrm{fm}$, we display also the circle with $R_{\mathrm{cr}}=32.7 \mathrm{fm}$, which determines the boundary (critical distance) between the subcritical and the supercritical regimes. Thus, we have the supercritical regime only during the period of time when the nuclei move from $R_{\text {cr }}$ to $R_{\text {min }}$ and back. This period of time decreases with increasing the collision energy $(E)$ and, therefore, with increasing the impact parameter $b$ (within the trajectories under consideration). This fact is clearly demonstrated in Fig. 4, which shows the supercritical regime duration as a function of $\eta=E / E_{0}$ at fixed $R_{\min }=$ $17.5 \mathrm{fm}$ (for all $b$ and $E$ ). For convenience, the figure also shows the scattering angles, $\theta$, which correspond to the given values of $\eta$. In the same figure, we present also the

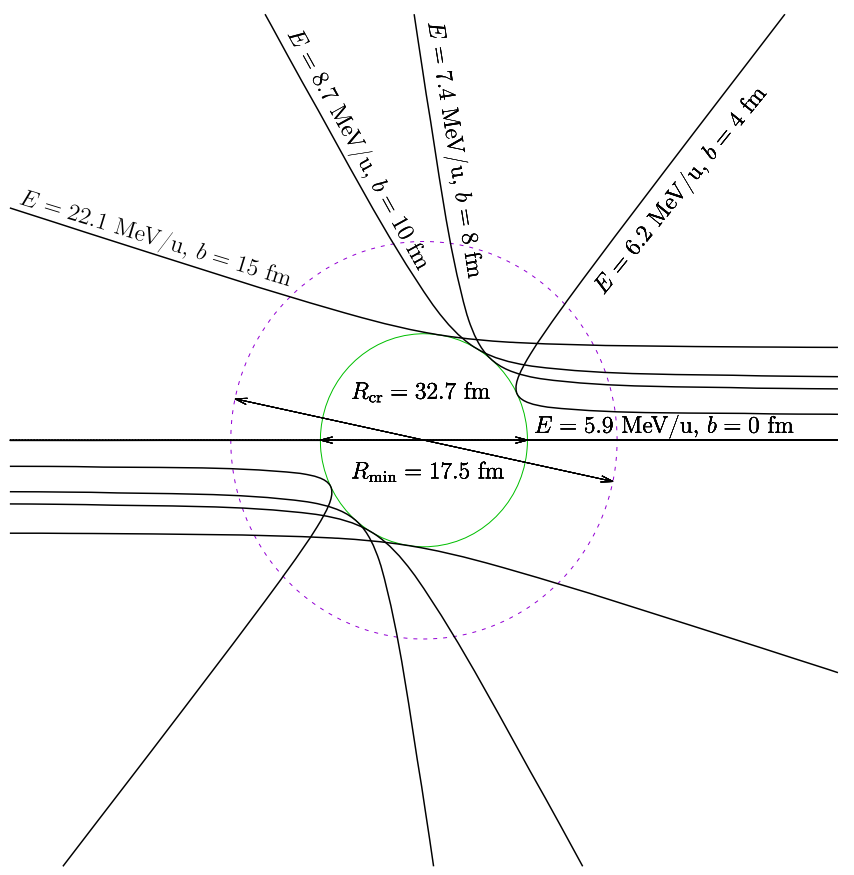

FIG. 3. The $\mathrm{U}^{92+}-\mathrm{U}^{92+}$ collision trajectories (in the center-ofmass frame) which correspond to the same minimal distance $R_{\min }=17.5 \mathrm{fm}$ for different impact parameters $b$ and, therefore, for different collision energies $E$. The energies $E$ denote the projectile kinetic energies in the rest frame of the target nucleus before the collision. The circle $R_{\min }=32.7 \mathrm{fm}$ defines the boundary (critical distance) between the subcritical and the supercritical regimes. 


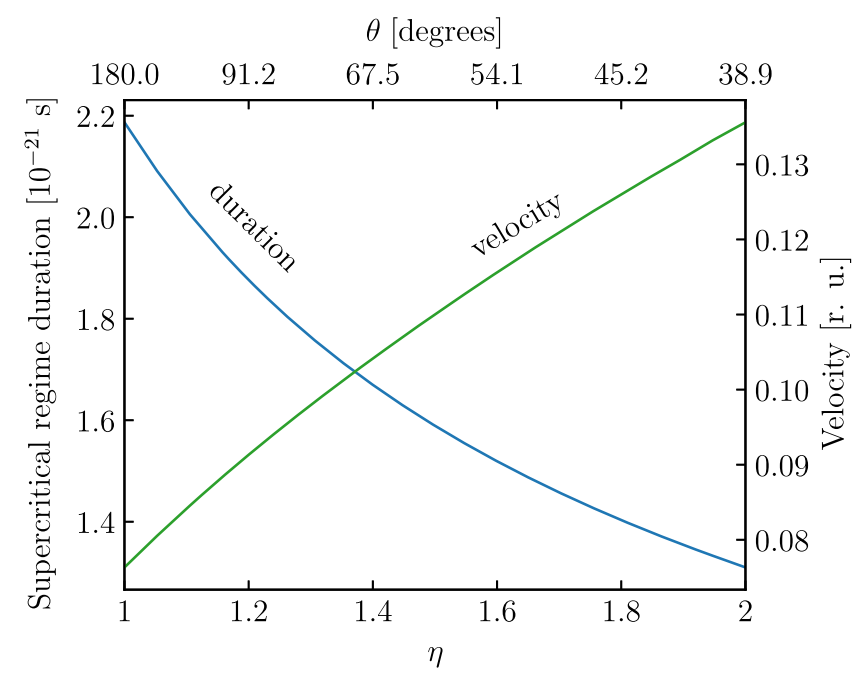

FIG. 4. The supercritical regime duration and the relative velocity of the nuclei at the $R=R_{\mathrm{cr}}$ in $\mathrm{U}^{92+}-\mathrm{U}^{92+}$ collisions at $R_{\min }=17.5 \mathrm{fm}$ (see Fig. 3) as functions of the collision energy, $\eta=E / E_{0}$, where $E_{0}$ is the head-on collision energy at the same $R_{\min }$. The scattering angles, $\theta$, which correspond to the given values of $\eta$, are also indicated.

relative velocity of the nuclei at the distance $R=R_{\mathrm{cr}}$ as a function of $E / E_{0}$. Opposite to the supercritical regime duration, this velocity increases with increasing $E / E_{0}$. It is clear that the dynamical pair creation must decrease monotonously with decreasing velocity (and, therefore, $E / E_{0}$ ) for a given $R_{\min }$. As to the spontaneous pair creation, it must increase monotonously with increasing the supercritical regime duration and, therefore, with decreasing $E / E_{0}$. It follows that any increase in the pair-production probability at $E / E_{0} \rightarrow 1$ for a given $R_{\min }$ should indicate the effect of the spontaneous pair creation, which takes place in the supercritical regime only.

\section{CALCULATIONS AND RESULTS}

The methods described in the previous section are employed for the calculations of the total pair-creation probabilities and positron spectra. The calculations are performed within the framework of the monopole approximation and are restricted to the $|\kappa|=1$ contributions. As is noted above, these contributions almost completely determine the values of interest and the difference between the monopole approximation results and the full two-center results does not exceed $10 \%$ in a wide interval of the scattering angles.

The positron-creation probabilities and positron spectra in heavy-ion collisions can be measured by requiring a coincidence between a positron event and scattered projectiles or recoil target nuclei at given angles $[58,59]$. If we neglect the nuclear-polarization effects and the oblate shape of the nuclei under consideration (e.g., uranium), there is a simple correspondence between the scattering angle and

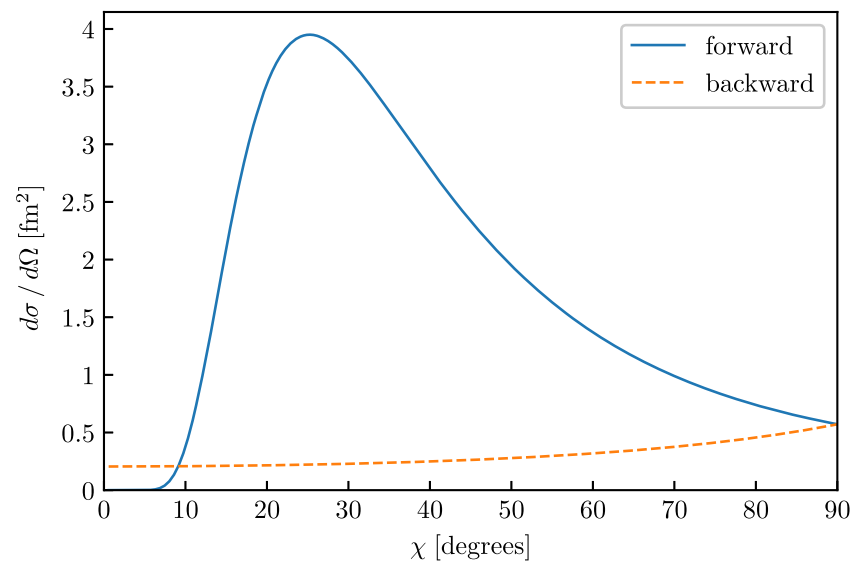

FIG. 5. The pair-creation cross sections as functions of $\chi=$ $180^{\circ}-\theta$ for the backward $\left(\theta>90^{\circ}\right)$ scattering trajectories and $\chi=\theta$ for the forward $\left(\theta<90^{\circ}\right)$ scattering trajectories.

the impact parameter. So, we can study the pair-creation probabilities as functions of the impact parameter. However, in case of fully symmetric collisions, one should keep in mind a problem of distinguishing the contributions for the angles $\theta$ and $180^{\circ}-\theta$, which correspond to different impact parameters. The calculations for the uraniumuranium collisions at the energies near the Coulomb barrier show that the pair creation from the backward scattering trajectories dominate over the pair creation from the related forward scattering trajectories in a rather small region: $\theta=180^{\circ}-172^{\circ}$. This can be seen from Fig. 5 which compares the corresponding pair-creation cross sections as functions of $\chi=180^{\circ}-\theta$ for the backward $\left(\theta>90^{\circ}\right)$ scattering trajectories and $\chi=\theta$ for the forward $\left(\theta<90^{\circ}\right)$ scattering trajectories. This problem does not appear for nonsymmetric collisions as well as for collisions of bare nuclei with neutral atoms, which will be briefly discussed at the end of this section. However, in what follows, for simplicity we will mainly consider the symmetric $\left(Z=Z_{1}=Z_{2}\right)$ collisions of bare nuclei assuming that, if necessary, the backward and forward trajectories can be distinguished by colliding different isotope beams. Some results for nonsymmetric collisions will be presented in Sec. III D.

\section{A. Pair-creation probabilities for symmetric collisions}

In Fig. 6 we present the results for the pair-creation probability in symmetric $\left(Z=Z_{1}=Z_{2}\right)$ collisions as a function of the parameter $\eta=E / E_{0}$, which is the ratio of the collision energy $E$ to the energy of the head-on collision $E_{0}$, and the nuclear charge number $Z$ at the same minimal internuclear distance $R_{\text {min }}$ (it means that only the trajectories as presented in Fig. 3 must be considered for each $R_{\min }$ ). The results are given for the values of $R_{\min }=17.5$, $25,50 \mathrm{fm}$, while the critical distance for the largest $Z$ presented $(Z=96)$ is about $48 \mathrm{fm}$. It means that in the case $R_{\min }=50 \mathrm{fm}$ for all $Z$ under consideration there is no contribution from the spontaneous pair creation, and, therefore, the pair creation is completely due to the 

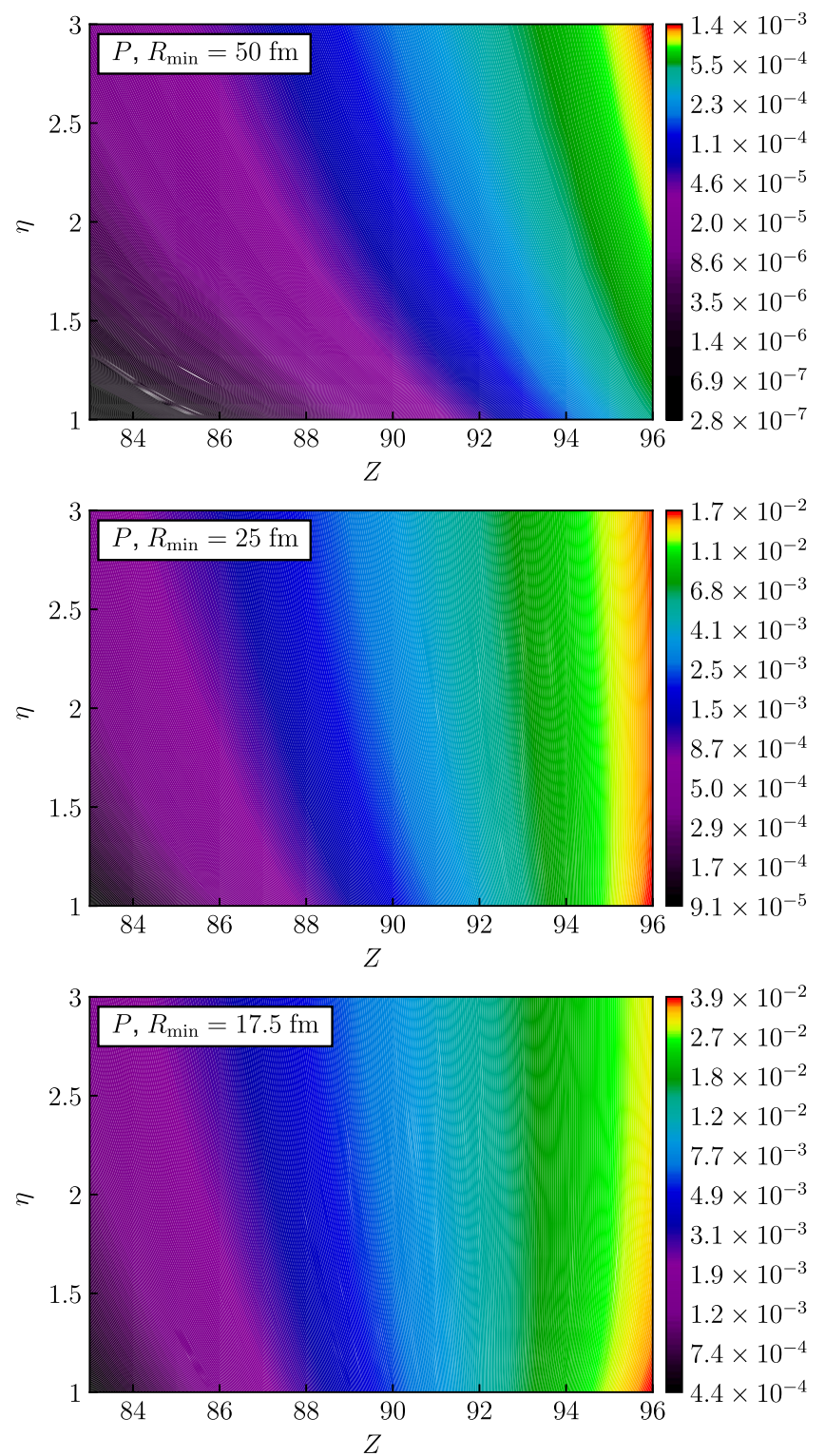

FIG. 6. The pair-creation probability in symmetric ( $Z=Z_{1}=Z_{2}$ ) collisions as a function of the parameter $\eta=E / E_{0}$, which is the ratio of the collision energy $E$ to the energy of the head-on collision $E_{0}$, and the nuclear charge number $Z$ at different minimal internuclear distance $R_{\min }$, which is the same within each image.

dynamical (induced) origin. As a result, with $R_{\min }=50 \mathrm{fm}$ for all $Z=84-96$ the pair-creation probabilities decrease monotonously when $\eta \rightarrow 1$. This is rather evident, since in the limit of adiabatically slow collisions the dynamical pair creation must be zero. The behavior of $P$ changes when the supercritical regime becomes available. For $R_{\min }=17.5 \mathrm{fm}$, in the subcritical regime $\left(Z<Z_{\text {cr }} / 2 \approx 87.5\right)$, the paircreation probability $P$ remains decreasing monotonously when $\eta \rightarrow 1$. However, the behavior of $P$ changes when the nuclear charge number $Z$ becomes sufficiently higher than the critical value. It can be seen that for such $Z$ the pair-creation probability $P$ increases at $\eta \rightarrow 1$. This is due to the spontaneous pair-creation mechanism which starts to work in the supercritical regime, while the dynamical pair creation decreases at $\eta \rightarrow 1$. To demonstrate this behavior in more detail, in Fig. 7 we display the $s(\kappa=-1)$ and $p_{1 / 2}(\kappa=1)$ contributions to the probability $P$ as functions of $\eta$ for $Z=$ 85-96 at $R_{\min }=17.5 \mathrm{fm}$ (similar graphs for $R_{\min }=16.5 \mathrm{fm}$ have been presented in Ref. [45]). As one can see from this figure, the $s$ contribution starts to increase significantly at $\eta \rightarrow 1$ already for $Z=92$, while the $p_{1 / 2}$ contribution remains decreasing at $\eta \rightarrow 1$ even at $Z=96$. In the case of $Z=92$, the increase of the $s$ contribution at $\eta \rightarrow 1$ is fully compensated by the decrease of the $p_{1 / 2}$ contribution, that leads to an almost constant value of their sum at small $\eta$. The different $\eta$ dependencies of the $s$ and $p_{1 / 2}$ contributions are explained by different values of $Z$ at which the $1 s$ and $2 p_{1 / 2}$ states enter the negative-energy continuum. For $R_{\min }=17.5 \mathrm{fm}$, this takes place at $Z=Z_{\mathrm{cr}}^{1 s} / 2 \equiv$ $Z_{\mathrm{cr}} / 2 \approx 87.5$ and $Z=Z_{\mathrm{cr}}^{2 p_{1 / 2}} / 2 \approx 95$ for $1 s$ and $2 p_{1 / 2}$ states, respectively (cf. Fig. 1).

Figure 8 displays the pair creation probabilities in symmetric $Z_{1}=Z_{2}=96$ collisions as a function of $R_{\min }$ and $\eta=E / E_{0}$, where $E_{0}$ corresponds to $R_{\min }$ under consideration. As one can see, the behavior of $P$ at $\eta \rightarrow 1$ changes when the minimal distance $R_{\min }$ becomes
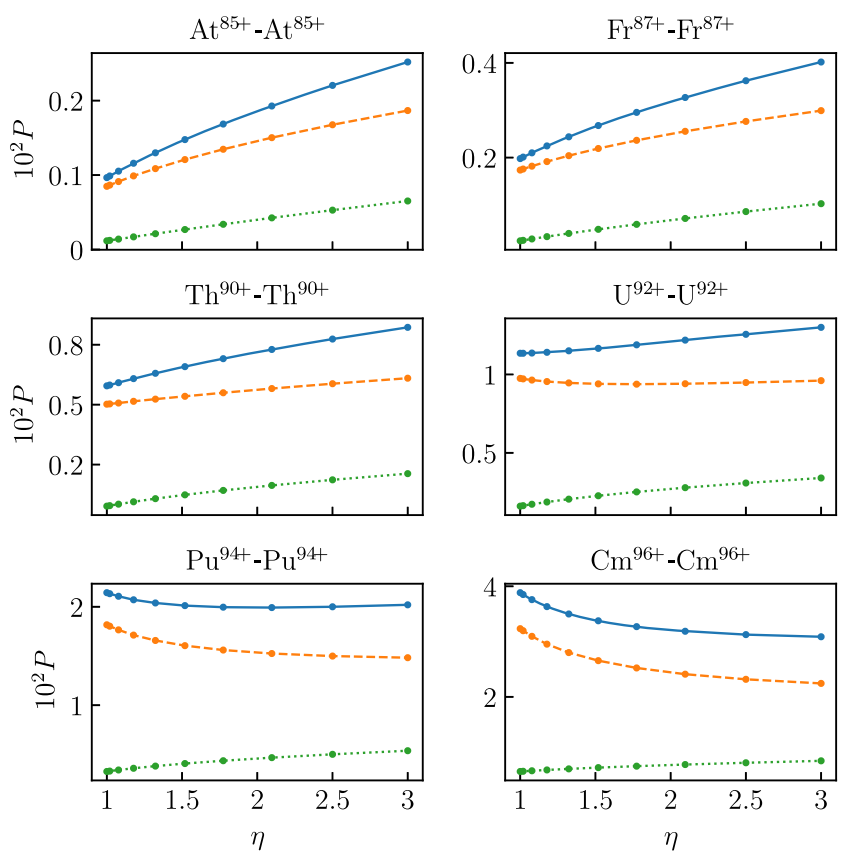

FIG. 7. The $s(\kappa=-1)$ and $p_{1 / 2}(\kappa=1)$ contributions to the pair-creation probability $P$ as functions of the parameter $\eta=E / E_{0}$, which is the ratio of the collision energy $E$ to the energy of the head-on collision $E_{0}$, at the same minimal internuclear distance $R_{\min }=17.5 \mathrm{fm}$. The $s$ and $p_{1 / 2}$ contributions are shown with the dashed (orange) and dotted (green) lines, respectively, while the total $P$ value is displayed by the solid (blue) line. 


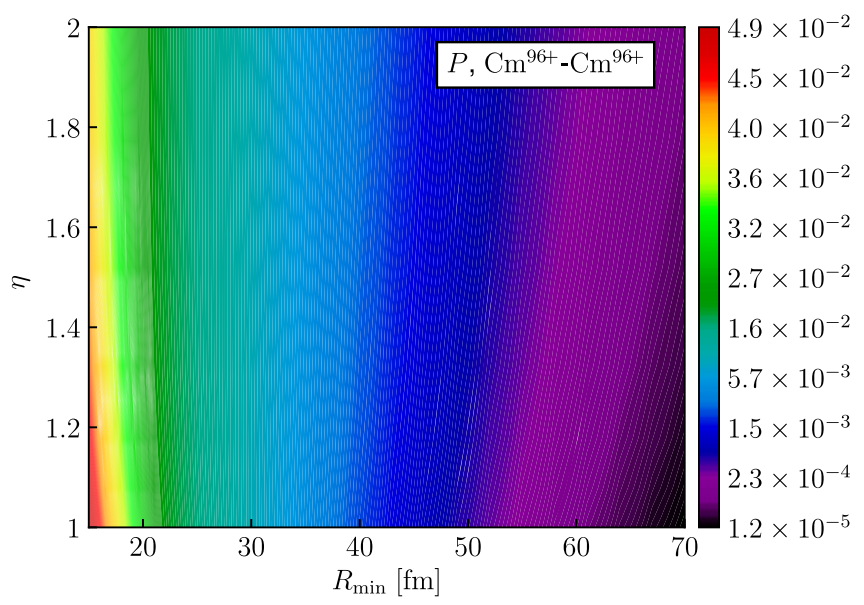

FIG. 8. The pair-creation probability in symmetric $Z_{1}=Z_{2}=$ 96 collisions as a function of the minimal internuclear distance $R_{\text {min }}$ and the parameter $\eta=E / E_{0}$, which is the ratio of the collision energy $E$ to the energy of the head-on collision at the same $R_{\min }$.

sufficiently smaller than the critical value, $R_{\mathrm{cr}} \approx 48 \mathrm{fm}$ for $Z=96$. In the subcritical region, $R_{\min }>R_{\mathrm{cr}}$, the pair creation is of pure dynamical origin and, therefore, can only decrease with decreasing $\eta$ (at a given value of $R_{\min }$ ). However, in the supercritical regime, $R_{\min }<R_{\mathrm{cr}}$, the spontaneous pair creation becomes possible and finally leads to an increase of $P$ when $\eta \rightarrow 1$.

Figure 9 presents the derivative of the pair-creation probability $P$ with respect to $\eta, d P / d \eta$, as a function of $Z$ and $R_{\min }$ at different scattering angles (in the center-ofmass frame): $\theta=60^{\circ}, 120^{\circ}, 180^{\circ}$. The black lines in this figure indicate the boundaries between the subcritical and the supercritical regimes. According to our discussion above, the pictures in Fig. 9 clearly demonstrate that the $d P / d \eta$ derivative, being strictly positive in the subcritical region, becomes negative when the parameters $Z$ and $R_{\min }$ enter deeply enough into the supercritical domain. Moreover, Fig. 9 shows that the transition from the subcritical to the supercritical region changes the behavior of $d P / d \eta$, starting with an increase when approaching the black line from the subcritical region and ending with a strong decrease after crossing this line. It can be seen that in the strong supercritical regime the $d P / d \eta$ derivative decreases in the directions which correspond to increasing $Z$ and decreasing $R_{\min }$. The profile $R_{\min }=16.5 \mathrm{fm}$, which demonstrates the change of the behavior of $d P / d \eta$ from the original increase at $Z<Z_{\mathrm{cr}} / 2$ to the subsequent decrease at $Z>Z_{\text {cr }} / 2$, has been presented for $\eta=1\left(\theta=180^{\circ}\right)$ in Ref. [45]. In Fig. 10 we present separately the $\kappa= \pm 1$ contributions to $d P / d \eta$ at $\eta=1\left(\theta=180^{\circ}\right)$ for the plane $R_{\min }=17.5 \mathrm{fm}$. It can be seen that, while the $\kappa=-1$ contribution has a maximum at $Z \approx 88$, the $\kappa=1$ contribution has it at $Z \approx 94.5$. This is due to a higher value of the critical charge for the $2 p_{1 / 2}$ state, $Z_{\mathrm{cr}}^{2 p_{1 / 2}} / 2 \approx 95$, compared
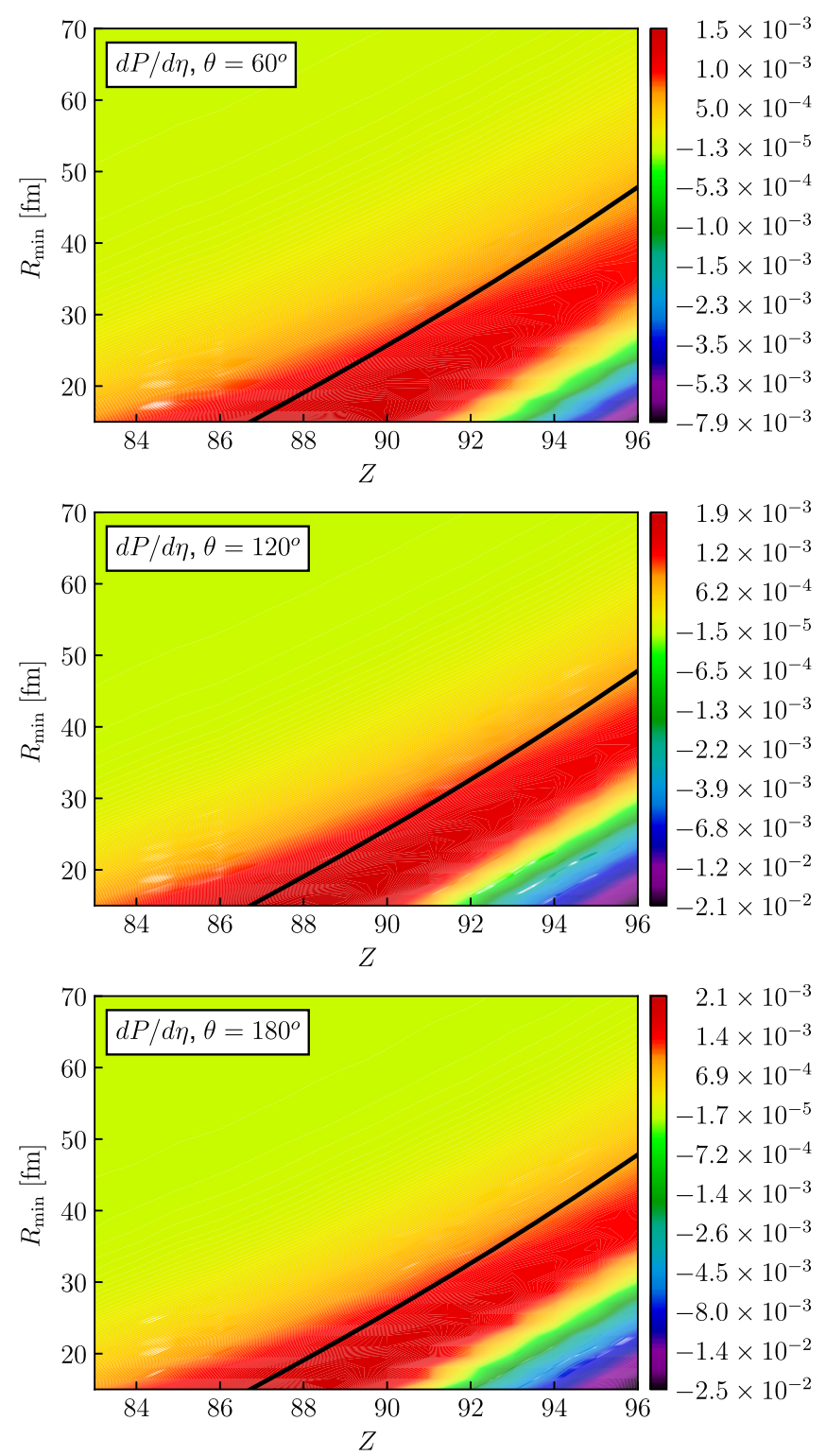

FIG. 9. The derivative of the pair-creation probability with respect to $\eta, d P / d \eta$, as a function of $Z=Z_{1}=Z_{2}$ and $R_{\min }$. The black line indicates the boundary between the subcritical and the supercritical regimes.

to the $1 s$ critical charge, $Z_{\mathrm{cr}}^{1 s} / 2 \approx 87.5$, (cf. Fig. 1 ). As a result, the maximum of the total contribution is shifted to $Z \approx 89$. The profile $Z=96$ at $\eta=1\left(\theta=180^{\circ}\right)$ in Fig. 9, which is separately presented in Fig. 11, shows a similar behavior of $d P / d \eta$, when $R_{\min }$ passes the critical values, $R_{\mathrm{cr}}^{1 s} \approx 48 \mathrm{fm}$ and $R_{\mathrm{cr}}^{2 p_{1 / 2}} \approx 20 \mathrm{fm}$ for $Z=96$.

\section{B. Positron spectra for symmetric collisions}

We have also calculated the positron energy distributions (in the center-of-mass frame) for the trajectories under consideration (Fig. 3). In Fig. 12 we present the total positron spectra for the symmetric collisions 


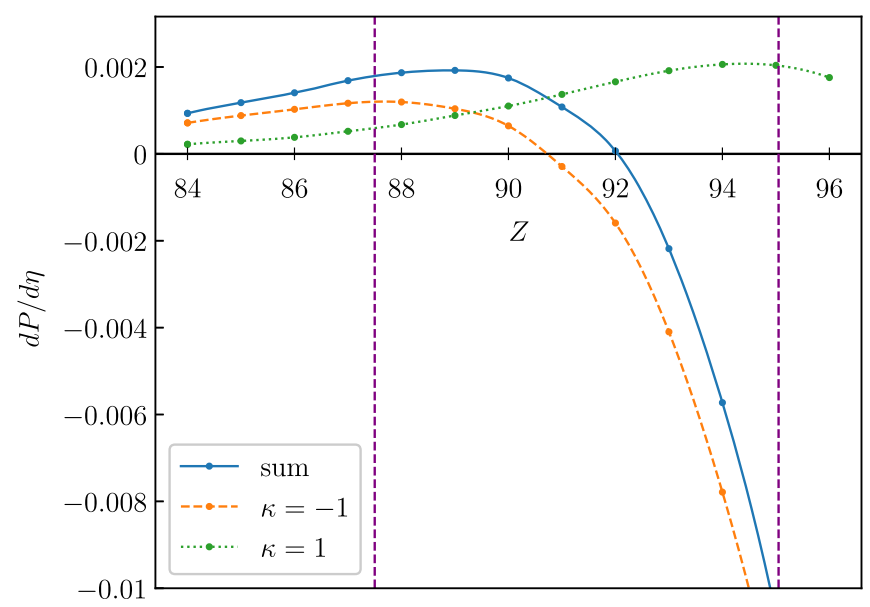

FIG. 10. The derivative of the pair-creation probability $d P / d \eta$ at $\eta=1 \quad\left(\theta=180^{\circ}\right)$ as a function of $Z=Z_{1}=Z_{2}$ for $R_{\min }=17.5 \mathrm{fm}$. The dashed vertical lines at $Z \approx 87.5$ and $Z \approx$ 95 indicate the boundaries between the subcritical and the supercritical regimes for the $1 s(\kappa=-1)$ and $2 p_{1 / 2}(\kappa=1)$ states, respectively.

with $Z=Z_{1}=Z_{2}=83-96$ at $R_{\min }=17.5 \mathrm{fm} \quad$ and $\eta=E / E_{0}=1.0,1.1,1.2$. In Figs. 13 and 14 we display separately the contributions to the positron spectra from the $s(\kappa=-1)$ and $p_{1 / 2}(\kappa=1)$ channels, respectively. As mentioned above, these two channels almost completely determine the total probability. The presented positron spectra demonstrate the well-known fact (see, e.g., Refs. [29,31]) that the emission of low-energy positrons is strongly suppressed by the repulsive interaction with the nuclei while at high energy the spectra fall off exponentially. In the case of $R_{\min }=17.5 \mathrm{fm}$, the supercritical

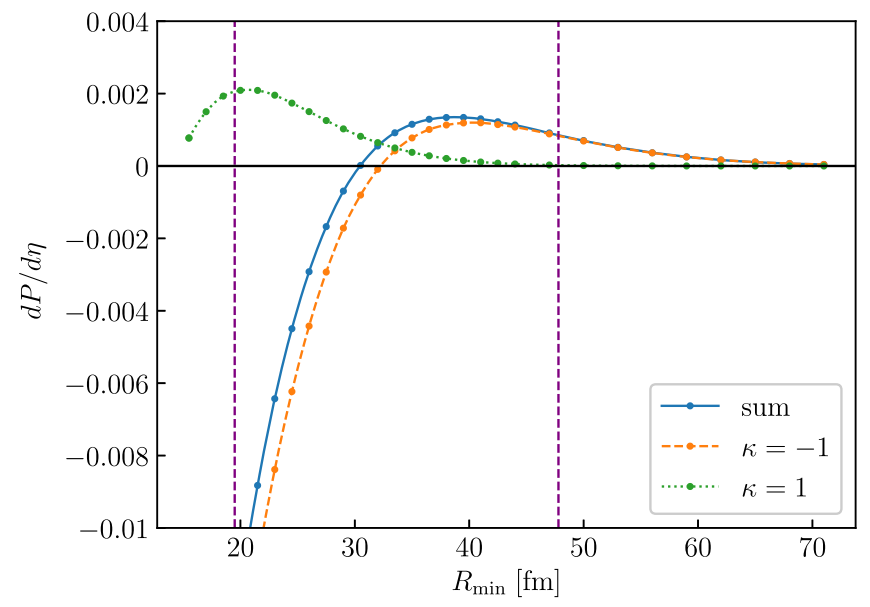

FIG. 11. The derivative of the pair-creation probability $d P / d \eta$ at $\eta=1\left(\theta=180^{\circ}\right)$ as a function of $R_{\min }$ for $Z_{1}=Z_{2}=96$. The dashed vertical lines at $R_{\min }=R_{\mathrm{cr}}^{1 s} \approx 48 \mathrm{fm}$ and $R_{\min }=R_{\mathrm{cr}}^{2 p_{1 / 2}} \approx$ $20 \mathrm{fm}$ indicate the boundaries between the subcritical and the supercritical regimes for the $1 s(\kappa=-1)$ and $2 p_{1 / 2}(\kappa=1)$ states, respectively. regime starts at $Z \approx 87.5$ and $Z \approx 95$ for the $1 s$ and $2 p_{1 / 2}$ states, respectively. As one can see from Figs. 12, 13 , and 14, in all cases the behavior of the positron spectra manifests a clear signature of the transition to the supercritical regime. Indeed, in the subcritical regime the positron spectrum curves at the energies near the maximum and higher are strictly ordered according to the $\eta$ values: the curve with a larger $\eta$ is strictly higher than the curve with a smaller $\eta$. This is due to the fact that the dynamical (induced) creation of positrons in general should increase with increasing the collision energy. In the supercritical regime, however, in the region near the maximum of the positron spectrum, the curves corresponding to smaller values of $\eta$ become higher than the ones with larger $\eta$, while at sufficiently large values of the positron energy the order of the curves remains the same as in the subcritical regime. This might be explained as follows. The spontaneous pair creation, which starts to work in the supercritical regime, is greater at lower values of $\eta$, which correspond to larger values of the supercritical time duration (Fig. 4). In addition, the spontaneous positrons are restricted to the lower part of the energy spectrum. For instance, in the case of the $\mathrm{U}^{92+}-\mathrm{U}^{92+}$ collision, the spontaneous positrons should be mainly limited to the energy range 0 to $600 \mathrm{keV}$. Indeed, let us consider first hypothetical collisions where the nuclei move adiabatically slowly along the actual trajectories [45,51]. In such collisions all created positrons are of pure spontaneous origin and, therefore, the energies of the emitted positrons are completely determined by the positions of the supercritical resonances. Table I presents these resonance energies, $\varepsilon_{\text {res }}$, and the related kinetic positron energies, $\varepsilon=\left|\varepsilon_{\text {res }}\right|-m_{e} c^{2}$, for the $\mathrm{U}^{92+}-\mathrm{U}^{92+}$ and $\mathrm{Cm}^{96+}-\mathrm{Cm}^{96+}$ systems at different internuclear distances. These energies have been obtained within the framework of the monopole approximation employing the complex rotation method [60-64]. The application of this method for the case of point nuclei leads to the results which are in good agreement with the values obtained by the use of related formulas from Ref. [65]. We note also that the corresponding calculations beyond the monopole approximation $[63,66]$ yield the resonance energies which are very close to the monopole-approximation ones. According to Table I, for the $\mathrm{U}^{92+}-\mathrm{U}^{92+}$ system the positron kinetic energies do not exceed $300 \mathrm{keV}$, while the related natural resonance widths do not exceed a few $\mathrm{keV}$ $[63,66]$. But in the real (nonadiabatic) collision the finite collision time yields a dynamical width, which is much larger than the natural resonance one. The dynamical width can be roughly estimated by the uncertainty principle $\Gamma_{\mathrm{dyn}} \sim \hbar / \tau_{\mathrm{col}}$, where $\tau_{\mathrm{col}}$ is determined as the supercritical time duration. With $\tau_{\text {col }} \sim 2 \times 10^{-21} \mathrm{~s}$ (see Fig. 4) one obtains $\Gamma_{\text {dyn }} \sim 300 \mathrm{keV}$, which restricts the energy of spontaneous positrons to the interval 0 to $600 \mathrm{keV}$. The large value of $\Gamma_{\mathrm{dyn}}$ prevents the existence of resonance structure in the positron spectra and smears 

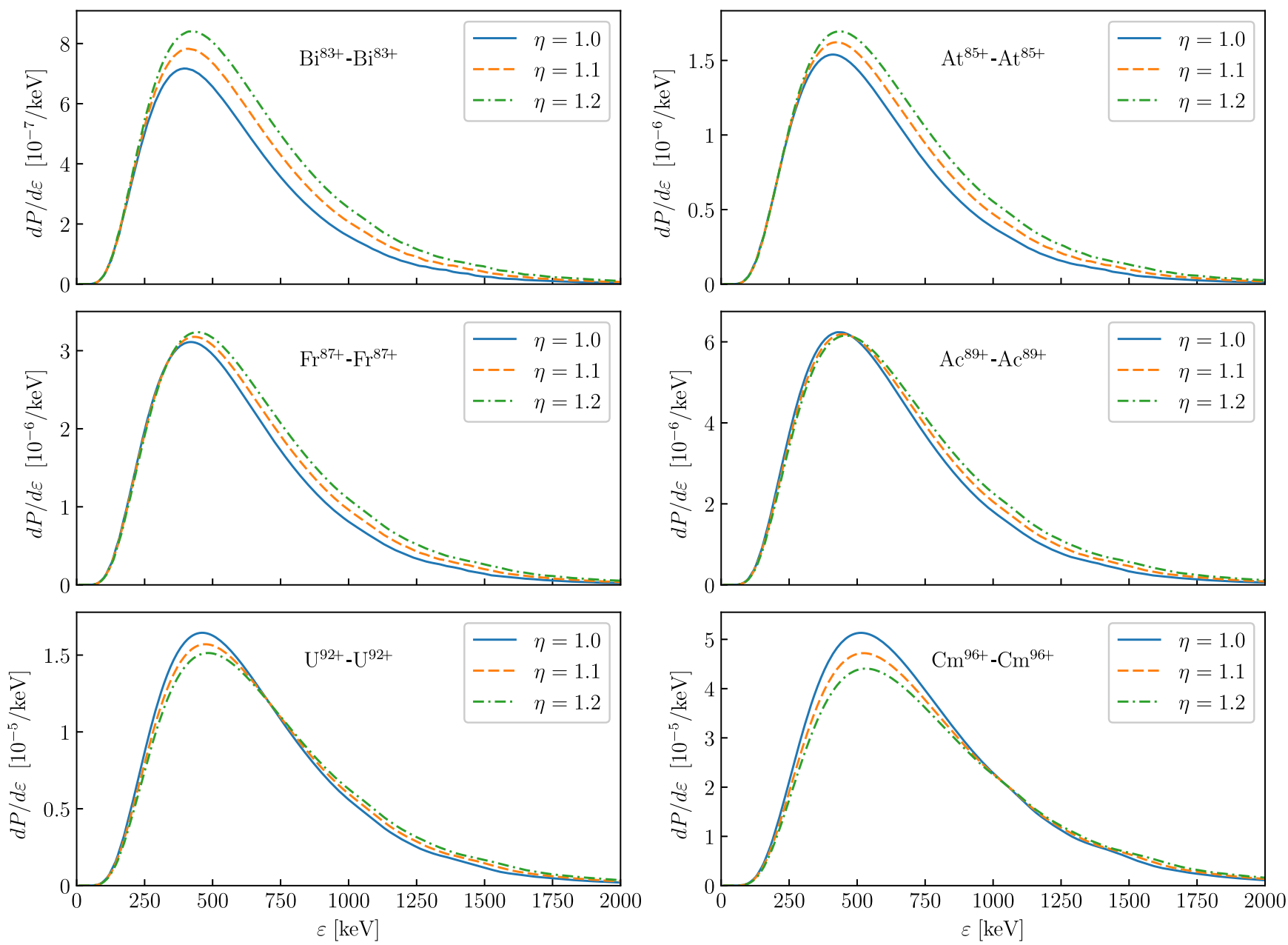

FIG. 12. The positron spectra for the symmetric collisions with $Z=Z_{1}=Z_{2}=83-96$ at $R_{\min }=17.5 \mathrm{fm}$ and $\eta=E / E_{0}=1.0,1.1$, 1.2 , which is the ratio of the collision energy $E$ to the energy of the head-on collision at the same $R_{\min }$.

the area, where the reverse order of the $\eta=1.0,1.1,1.2$ curves takes place, compared to the subcritical case. In addition, the strong superposition of the dynamical and spontaneous pair-creation mechanisms in the real collisions makes the transition from the subcritical to the supercritical mode quite smooth. However, the qualitative changes in the positron spectra, which become especially convincing when comparing the subcritical $\mathrm{Bi}^{83+}-\mathrm{Bi}^{83+}$ and supercritical $\mathrm{Cm}^{96+}-\mathrm{Cm}^{96+}$ collisions, must be considered as a conclusive proof of the access to the supercritical mode. This statement is also confirmed by Fig. 15, where the positron spectra for the $\mathrm{U}^{92+}-\mathrm{U}^{92+}$ collision at different $R_{\min }$ are presented. Again, the transition from the subcritical to the supercritical mode $\left(R_{\mathrm{cr}}=32.7 \mathrm{fm}\right)$ changes the order of the curves near the maximum.

As one can see from Figs. 12, 13, 14, and 15, the changes in the positron spectra caused by the transition to the supercritical mode are mainly concentrated at the energies around the spectrum maximum and lower, where the spontaneous pair creation takes place. This fact can be used to quantify the signature of the transition to the supercritical mode in terms an integral characteristic of the positron spectrum which accounts only for a part of it instead of the total probability. It is natural to restrict this part to the region, where the changes of interest take place. To this end, we introduce a partial probability $P_{x}$, which is defined as shown in Fig. 16. This means that the probability $P_{x}$ accounts only for the positrons with the energies not exceeding the abscissa value corresponding to the point $d P / d \varepsilon=(1-x)(d P / d \varepsilon)_{\max }$ on the right-hand side from the positron-spectrum maximum at $\eta=1\left(\theta=180^{\circ}\right)$. The parameter $x$ should be chosen to cover the area which includes the positrons with the energies within the range $\Gamma_{\text {dyn }}$ from the resonance energy. As discussed above, in the case of the $\mathrm{U}^{92+}-\mathrm{U}^{92+}$ collision the energies of the spontaneous positrons are mainly limited by about $600 \mathrm{keV}$. It follows that the parameter $x$ should be chosen not less than $x \approx 0.1$, which corresponds to the inclusion of about $50 \%$ of the total probability.

In Fig. 17 we display the derivative $d \log P_{x} / d \eta=$ $\left(1 / P_{x}\right) d P_{x} / d \eta$ at $\eta=1\left(\theta=180^{\circ}\right)$ for $x=0.1,0.25$, 

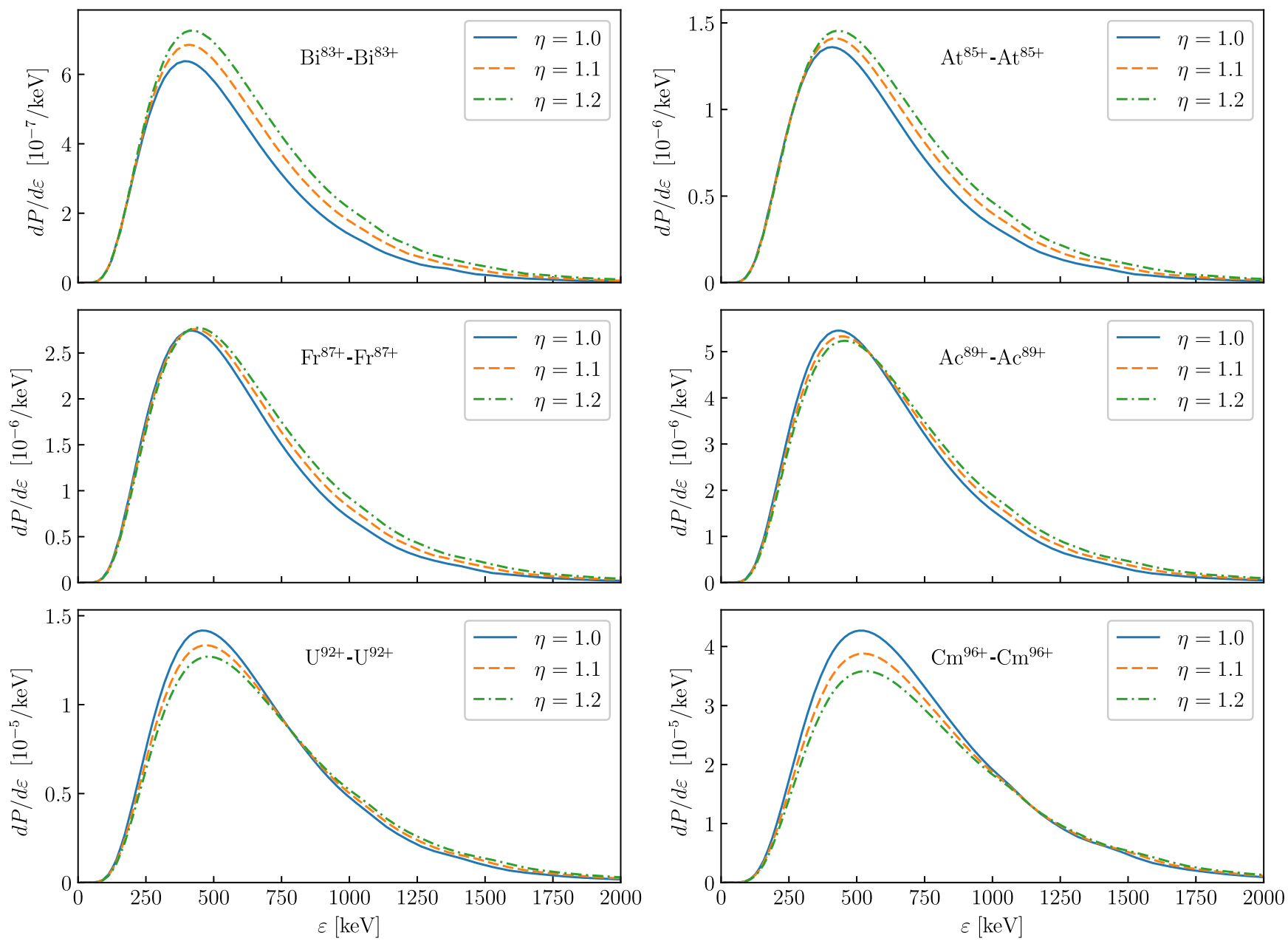

FIG. 13. The $s$-wave $(\kappa=-1)$ contribution to the positron spectra for the symmetric collisions with $Z=Z_{1}=Z_{2}=83-96$ at $R_{\min }=17.5 \mathrm{fm}$ and $\eta=E / E_{0}=1.0,1.1,1.2$, which is the ratio of the collision energy $E$ to the energy of the head-on collision at the same $R_{\min }$.

$0.5,1.0$ in the symmetric $\left(Z=Z_{1}=Z_{2}\right)$ collisions in the range $Z=84-92$. As one can see from the figure, the use of $d \log P_{x} /\left.d \eta\right|_{\eta=1}$ at $x=0.1,0.25$, and even at $x=0.5$ (instead of $x=1$ corresponding to the total probability) leads to a strong increase of the effect we are interested in. Indeed, in the case of the $\mathrm{U}^{92+}-\mathrm{U}^{92+}$ collision, the derivative $d \log P_{x} /\left.d \eta\right|_{\eta=1}$ amounts to about -0.6 at $x=0.1,-0.5$ at $x=0.25$, and -0.4 at $x=0.5$, while for the total probability $\left(d \log P_{x=1} /\left.d \eta\right|_{\eta=1}\right)$ it is almost zero. This means taking into account only a part of the positron spectrum, as defined above, allows to get a much stronger evidence of the transition to the supercritical mode. This statement is supported by Fig. 18, where we present the ratio of $P_{x}$ evaluated at different $\eta$ to its value at $\eta=1\left(\theta=180^{\circ}\right)$ as a function of $Z=Z_{1}=Z_{2}$. The transition from the region $P_{x}(\eta) / P_{x}(1)>1$ to the region $P_{x}(\eta) / P_{x}(1)<1$ indicates the transition from the subcritical to the supercritical mode. It can be seen that for $x=0.1$, $0.25,0.5$ this transition takes place at lower $Z$ values than for $x=1$ (total probability). In Fig. 19, we present the same ratio, $P_{x}(\eta) / P_{x}(1)$, for the $\mathrm{U}^{92+}-\mathrm{U}^{92+}$ collision as a function of $R_{\min }$. Again, this figure clearly confirms the advantage of studying a part of the spectrum compared to the total one and makes the uranium-uranium collisions very promising for observing the vacuum decay.

\section{Pair-creation probabilities and positron spectra for nonsymmetric collisions}

All the above studies can be directly extended to nonsymmetric collisions. In Fig. 20 we present the paircreation probability in non-symmetric $\mathrm{U}^{92+}-\mathrm{Cm}^{96+}$ collisions as a function of the minimal internuclear distance $R_{\min }$ and the parameter $\eta=E / E_{0}$. As in the case of the symmetric $\mathrm{Cm}^{96+}-\mathrm{Cm}^{96+}$ collisions (see Fig. 8), the paircreation probability $P$ starts to increase with decreasing $\eta$ when the system enters deeply enough into the supercritical regime, which means that $R_{\min }$ becomes significantly smaller than the critical value, $R_{\mathrm{cr}} \approx 40 \mathrm{fm}$. In contrast, in the subcritical region $P$ decreases with decreasing $\eta$ (at a 

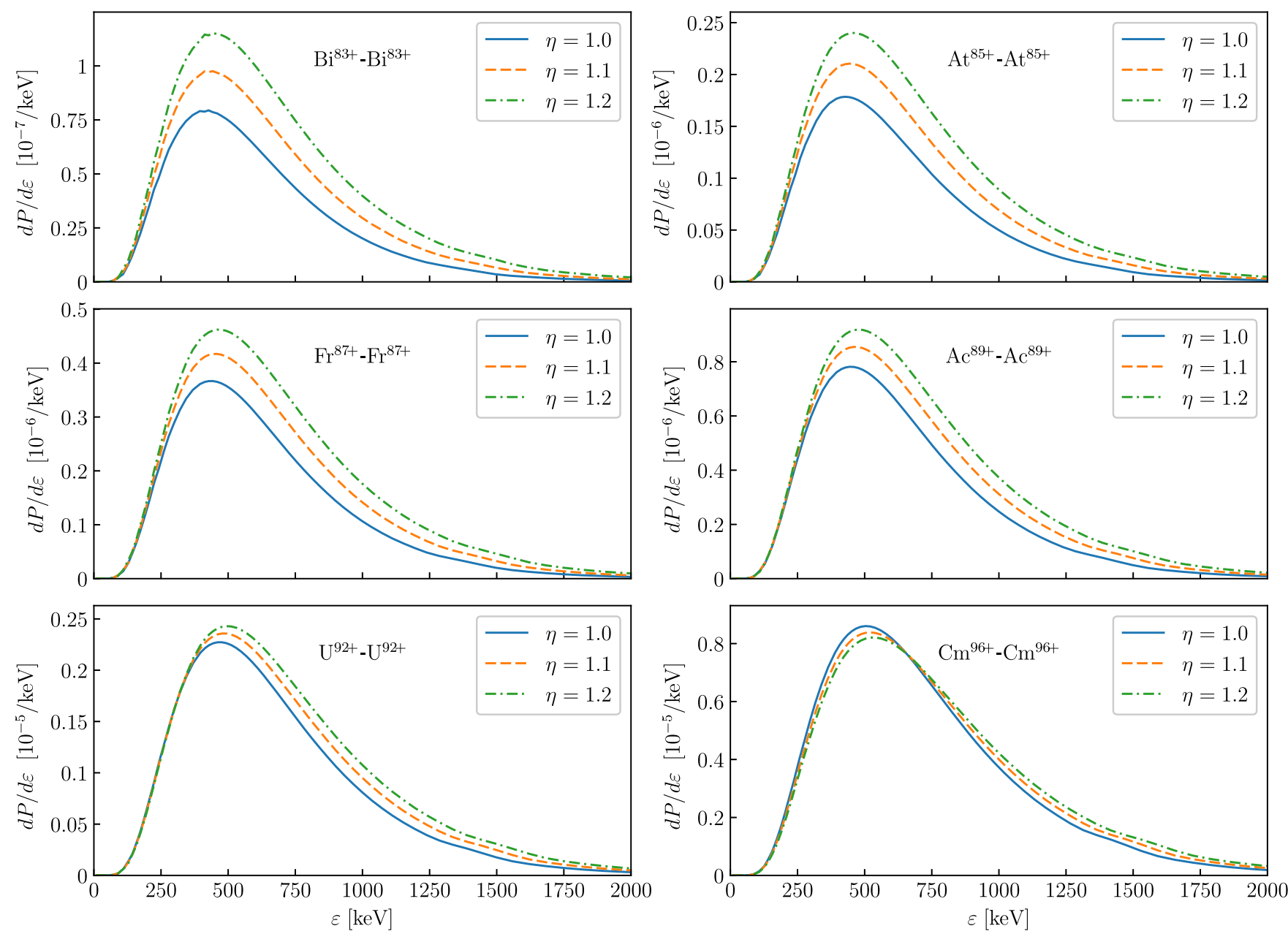

FIG. 14. The $p_{1 / 2}$-wave $(\kappa=1)$ contribution to the positron spectra for the symmetric collisions with $Z=Z_{1}=Z_{2}=83-96$ at $R_{\min }=17.5 \mathrm{fm}$ and $\eta=E / E_{0}=1.0,1.1,1.2$, which is the ratio of the collision energy $E$ to the energy of the head-on collision at the same $R_{\min }$.

given value of $\left.R_{\min }\right)$. In Fig. 21 we present the positron spectra for nonsymmetric $\mathrm{Po}^{84+}-\mathrm{Ra}^{88+}, \mathrm{Ra}^{88+}-\mathrm{U}^{92+}$, and $\mathrm{U}^{92+}-\mathrm{Cm}^{96+}$ collisions. As in the case of the symmetric collisions, the behavior of the positron spectra indicates a clear signature of the transition to the supercritical regime.

\section{Pair creation in collisions of bare nuclei with neutral atoms}

In the previous sections, we studied the pair creation in collisions of bare nuclei only. In this section, we will consider whether it is possible in principle to extend these studies to collisions of bare nuclei with neutral atoms. While the direct calculation of the pair production in such collisions requires development of special methods that are beyond the scope of this work, some rough estimates can be made by calculating the probability of a vacancy in the $1 \sigma$ state of a quasimolecule formed in such a collision. This is due to the fact that, in accordance with the results for collisions of bare nuclei, the main contribution to the pair creation is given by the process with the electron captured into the lowest-energy bound state [33,42-44]. Therefore, the pair-creation probability is mainly defined by availability of a vacancy in the $1 \sigma$ level when the nuclei approach each other. In this section, we evaluate the probability of a vacancy in the $1 \sigma$ state at the critical distance for the $\mathrm{U}-\mathrm{U}^{92+}$ and $\mathrm{Cm}-$ $\mathrm{U}^{92+}$ collisions using the two-center method developed in Ref. [38].

The quantum dynamics of electrons in the collision of the neutral $\mathrm{U}$ and $\mathrm{Cm}$ atoms with the bare $\mathrm{U}$ nucleus is described by the time-dependent Dirac equation with the two-center potential given by a sum of the potential of the neutral atom and the Coulomb potential of the bare nucleus. The potential of the neutral atom is defined by the density functional theory (DFT) in the local density approximation (LDA) using the Perdew-Zunger (PZ) parametrization [67] (see Ref. [38] for details). The basis is formed by the Dirac-Fock (DF) orbitals for the occupied 

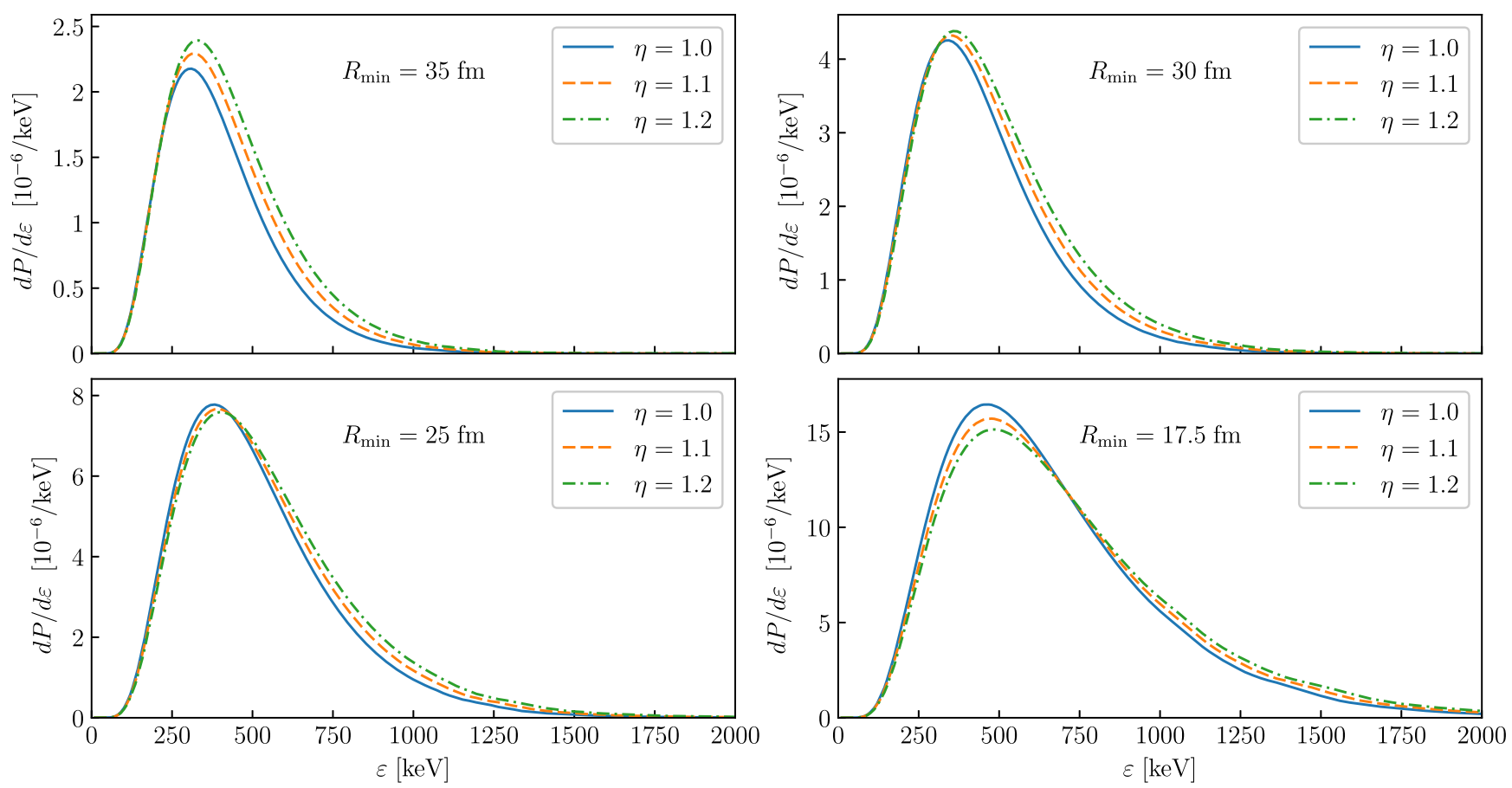

FIG. 15. The positron spectra in the $\mathrm{U}^{92+}-\mathrm{U}^{92+}$ collision at different $R_{\min }$ and $\eta=E / E_{0}$, which is the ratio of the collision energy $E$ to the energy of the head-on collision at the same $R_{\min }$.

states and by the Dirac-Fock-Sturm (DFS) orbitals for the vacant (virtual) states, localized at each of the two centers (see Refs. [37,38] and references therein). The DF and DFS orbitals are obtained in the central field approximation by numerical solution of the related integrodifferential DF and DFS equations. The radial DF and DFS Hamiltonians describe the atom (ion) in the external field induced by the other ion (atom). This external field is defined by the DFT method in the LDA approximation with the PZ parametrization. To the leading order, the external potential effect can be taken into account by including the external potential within the monopole approximation, when only the spherically-symmetric part of the reexpansion of the potential of the other atom (ion) is

TABLE I. Supercritical resonance energies $\varepsilon_{\text {res }}$ and related kinetic positron energies $\varepsilon=\left|\varepsilon_{\text {res }}\right|-m_{e} c^{2}$ for the $\mathrm{U}^{92+}-\mathrm{U}^{92+}$ and $\mathrm{Cm}^{96+}-\mathrm{Cm}^{96+}$ systems at different internuclear distances $R$ calculated within the framework of the monopole approximation.

\begin{tabular}{|c|c|c|c|c|c|c|}
\hline \multirow[b]{3}{*}{$R(\mathrm{fm})$} & \multirow{2}{*}{\multicolumn{2}{|c|}{$\begin{array}{l}\mathrm{U}^{92+}-\mathrm{U}^{92+} \\
s \text { resonance }\end{array}$}} & \multicolumn{4}{|c|}{$\mathrm{Cm}^{96+}-\mathrm{Cm}^{96+}$} \\
\hline & & & \multicolumn{2}{|c|}{$s$ resonance } & \multicolumn{2}{|c|}{$p_{1 / 2}$ resonance } \\
\hline & $\varepsilon_{\mathrm{res}} / m_{e} c^{2}$ & $\varepsilon(\mathrm{keV})$ & $\varepsilon_{\mathrm{res}} / m_{e} c^{2}$ & $\varepsilon(\mathrm{keV})$ & $\varepsilon_{\mathrm{res}} / m_{e} c^{2}$ & $\varepsilon(\mathrm{keV})$ \\
\hline 16 & -1.582 & 298 & -2.154 & 589 & -1.228 & 116 \\
\hline 18 & -1.482 & 246 & -2.011 & 517 & -1.092 & 47 \\
\hline 20 & -1.392 & 200 & -1.885 & 452 & & \\
\hline 22 & -1.311 & 159 & -1.773 & 395 & & \\
\hline 24 & -1.239 & 122 & -1.673 & 344 & & \\
\hline 26 & -1.174 & 89 & -1.584 & 298 & & \\
\hline
\end{tabular}

taken into account. It should be noted that the basis constructed in this way changes as the internuclear distance changes. In other words, this basis depends on the time and the corresponding time derivatives must be added

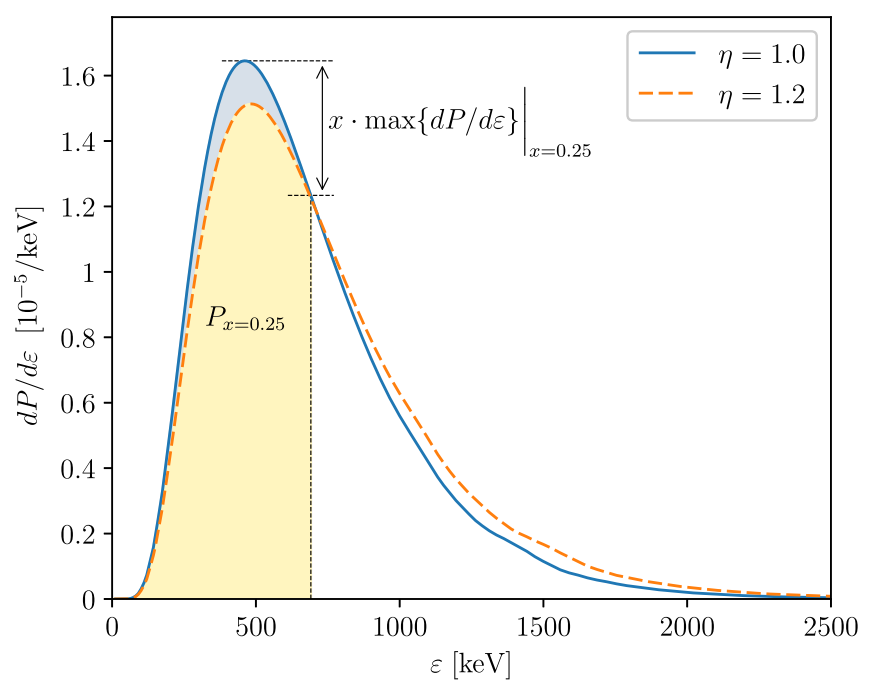

FIG. 16. The definition of the partial probability $P_{x}$ for $x=$ 0.25 in the case of the $\mathrm{U}^{92+}-\mathrm{U}^{92+}$ collision. $P_{x}$ accounts only for the positrons with the energies not exceeding the abscissa value corresponding to the point $d P / d \varepsilon=(1-x)(d P / d \varepsilon)_{\max }$ on the right-hand side from the positron-spectrum maximum at $\eta=1 \quad\left(\theta=180^{\circ}\right)$. The area shaded yellow corresponds to $P_{x}(\eta=1.2)$, while $P_{x}(\eta=1)$ incorporates the areas shaded yellow and blue. 


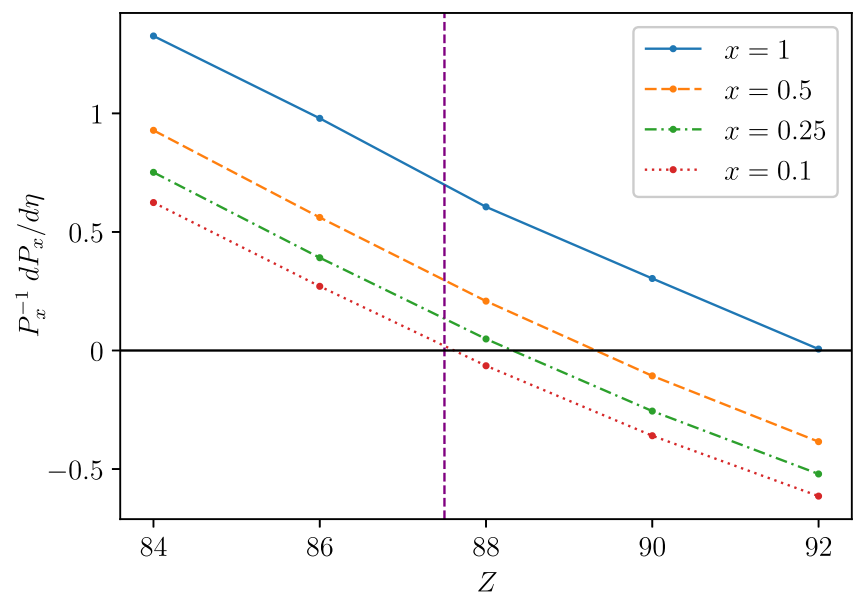

FIG. 17. The derivative $d \log P_{x} / d \eta=\left(1 / P_{x}\right) d P_{x} / d \eta$ at $\eta=1$ $\left(\theta=180^{\circ}\right)$ for different $x$ in the symmetric $\left(Z=Z_{1}=Z_{2}\right)$ collisions. The dashed vertical line at $Z \approx 87.5$ indicates the boundary between the subcritical and the supercritical regime.

to the Hamiltonian matrix [37]. The basis expansion coefficients of a set of one electron time-dependent wave functions $\psi_{i}(t)$ are defined using the direct evolution (exponential) operator $\hat{U}$ method [37]. These coefficients are represented by the columns of the $U$ evolution matrix (the matrix of the operator $\hat{U}$ ).

The probability of pair creation with electron captured into a vacancy at the quasimolecular $1 \sigma$ level can be roughly estimated as

$$
P(C)=2 P(C / \bar{A} B) \cdot K_{\mathrm{vac}},
$$

where $P(C / \bar{A} B)$ is the conditional probability of a pair being created and the vacancy being filled, provided that
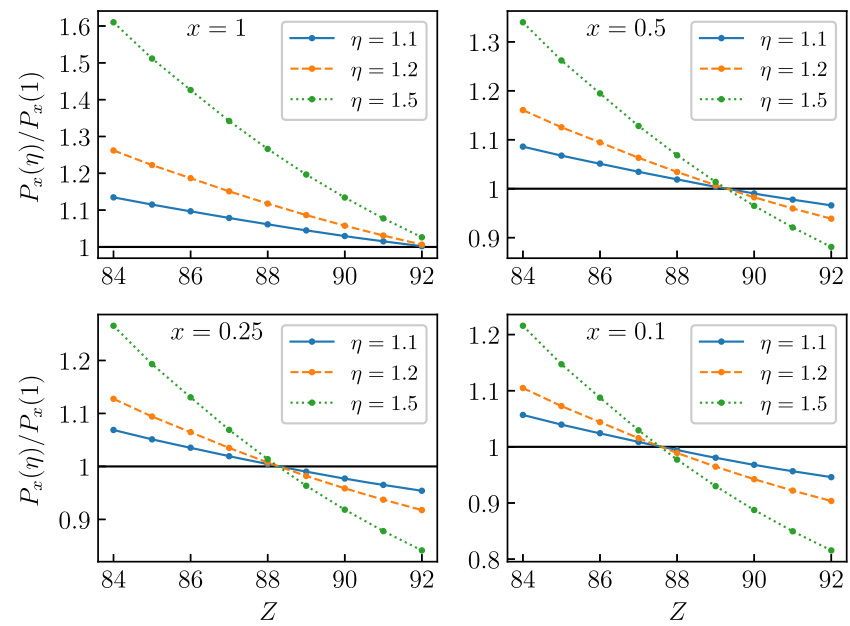

FIG. 18. The ratio of $P_{x}$ evaluated at different $\eta$ to its value at $\eta=1\left(\theta=180^{\circ}\right)$ as a function of $Z=Z_{1}=Z_{2}$. The transition from the region $P_{x}(\eta) / P_{x}(1)>1$ to the region $P_{x}(\eta) / P_{x}(1)<1$ indicates the transition from the subcritical to the supercritical mode.
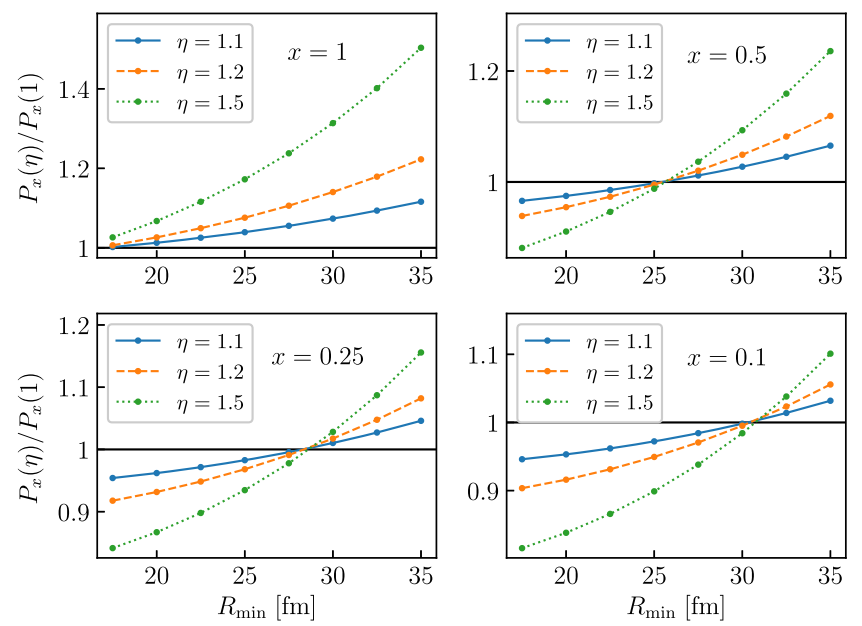

FIG. 19. The ratio of $P_{x}$ evaluated at different $\eta$ to its value at $\eta=1\left(\theta=180^{\circ}\right)$ as a function of $R_{\min }$ for the $\mathrm{U}^{92+}-\mathrm{U}^{92+}$ collision. The transition from the region $P_{x}(\eta) / P_{x}(1)>1$ to the region $P_{x}(\eta) / P_{x}(1)<1$ indicates the transition from the subcritical to the supercritical mode $\left(R_{\mathrm{cr}}=32.7 \mathrm{fm}\right)$.

there is exactly one $1 \sigma_{1 / 2}$ vacancy (event $\bar{A}$ ) and the state $1 \sigma_{-1 / 2}$ is occupied (event B). The coefficient $K_{\text {vac }}$ takes into account the probability of filling one or two vacancies and the factor 2 accounts for two values of the angular momentum projection $\mu= \pm 1 / 2$ (see the Appendix for details).

To calculate the probability of a vacancy in the $1 \sigma$ level of the Hamiltonian of a quasimolecule at a given time we use the approach which is described in the Appendix. This approach is equivalent to a method which was previously used for the evaluation of inclusive probabilities (see Refs. $[68,69]$ and references therein).

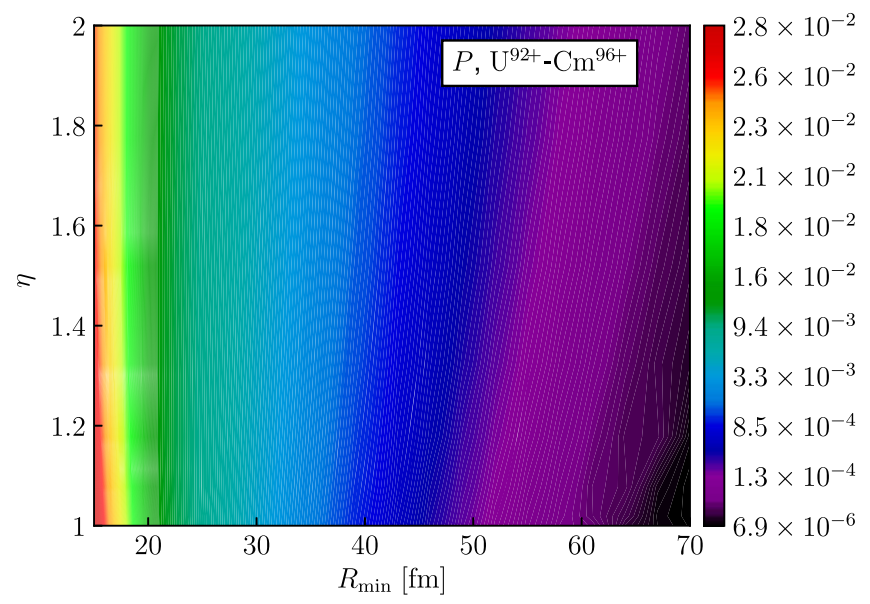

FIG. 20. The pair-creation probability in nonsymmetric $\mathrm{U}^{92+}-$ $\mathrm{Cm}^{96+}$ collisions as a function of the minimal internuclear distance $R_{\min }$ and the parameter $\eta=E / E_{0}$, which is the ratio of the collision energy $E$ to the energy of the head-on collision at the same $R_{\min }$. 

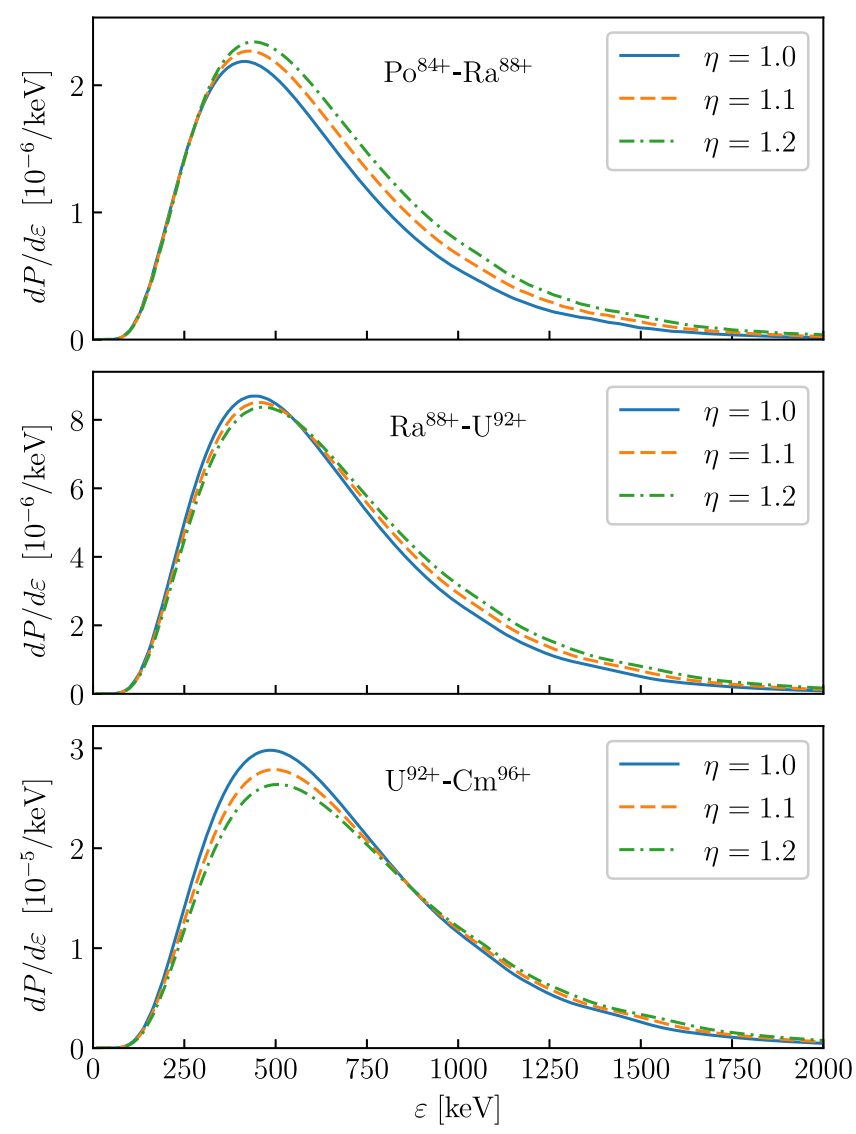

FIG. 21. The positron spectra for nonsymmetric $\mathrm{Po}^{84+}-\mathrm{Ra}^{88+}$ $\mathrm{Ra}^{88+}-\mathrm{U}^{92+}$, and $\mathrm{U}^{92+}-\mathrm{Cm}^{96+}$ collisions at $R_{\min }=17.5 \mathrm{fm}$ and $\eta=E / E_{0}=1.0,1.1,1.2$.

We define the time-dependent one-particle density operator $\hat{\rho}(t)$ and one-hole density operator $\hat{\bar{\rho}}(t)$

$$
\hat{\rho}(t)=\sum_{i} q_{i}\left|\psi_{i}(t)\right\rangle\left\langle\psi_{i}(t)\right|, \quad \hat{\bar{\rho}}(t)=\hat{I}-\hat{\rho}(t),
$$

where $q_{i}=0,1$ is the number of electrons on a spin-orbital $\psi_{i}$, which is defined by the initial condition. The inclusive probability $P_{n}$ of finding $n$ states occupied in an $N$ electron system while the remaining $N-n$ electrons are not detected is given by the determinant of an $n \times n$ matrix $\gamma$ constructed from the one-electron density matrix [68]

$$
P_{n}=\operatorname{det} \gamma, \quad \gamma_{k j}=\left\langle\varphi_{k}|\hat{\rho}| \varphi_{j}\right\rangle,
$$

where $\varphi_{k}$ are the stationary wave functions of the Hamiltonian at a given time, and $k, j=1, \ldots, n$. Similarly, the probability $\bar{P}_{n}$ of finding $n$ states unoccupied is determined by

$$
\bar{P}_{n}=\operatorname{det} \bar{\gamma}, \quad \bar{\gamma}_{k j}=\delta_{k j}-\gamma_{k j} .
$$

In this paper, we investigated the probability $\bar{P}_{n}$ of finding two vacancies $(n=2)$. As a result, the coefficient $K_{\text {vac }}$ has

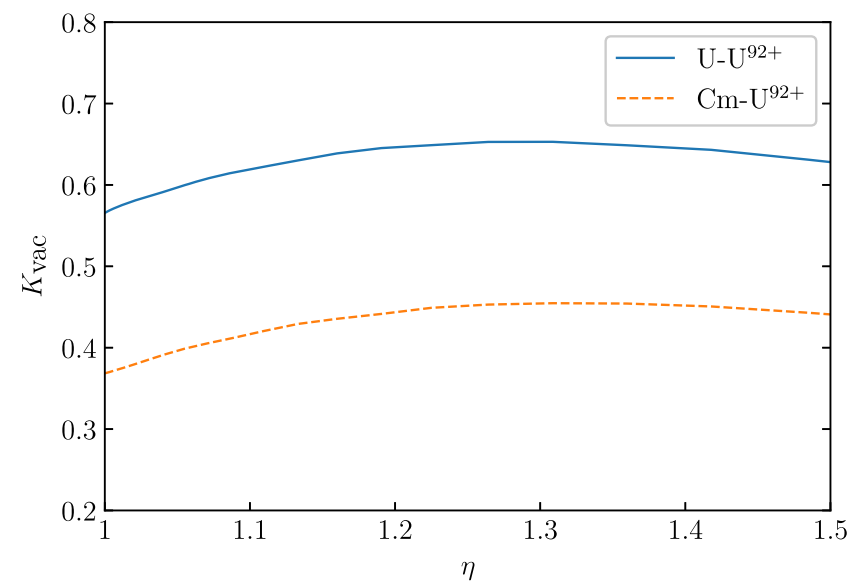

FIG. 22. The coefficient $K_{\text {vac }}$ evaluated by Eq. (34) for the $1 \sigma$ level at the critical internuclear distances, $27.0 \mathrm{fm}$ and $34.8 \mathrm{fm}$ for the $\mathrm{U}-\mathrm{U}^{92+}$ and $\mathrm{Cm}-\mathrm{U}^{92+}$ collisions, respectively, as a function of the collision energy, $\eta=E / E_{0}$, where $E_{0}$ is the head-on collision energy. The calculations are performed for the Rutherford trajectories with $R_{\min }=17.5 \mathrm{fm}$.

been calculated using the following expression (see the Appendix)

$$
K_{\mathrm{vac}}=1-\gamma_{11} \text {. }
$$

As the occupied $q_{i}=1$ spin orbitals $\psi_{i}(t)$, we consider all single-electron states of the ground-state configuration of the neutral atom $\mathrm{U}$ or $\mathrm{Cm}$ and all states of the negativeenergy Dirac continuum. All the positive-energy states of the bare uranium nucleus are considered as unoccupied. The occupation of single-electron states of the negativeenergy spectrum corresponds to the Dirac picture and takes into account the Pauli exclusion principle and pair production processes. By means of the Eq. (34) we calculated the $1 \sigma$ vacancy probabilities for the Rutherford trajectories presented in Fig. $3\left(R_{\min }=17.5 \mathrm{fm}\right)$. In Fig. 22 we present the coefficient $K_{\mathrm{vac}}$ at the critical internuclear distance, which equals to $27.0 \mathrm{fm}$ and $34.8 \mathrm{fm}$ for the $\mathrm{U}-\mathrm{U}^{92+}$ and $\mathrm{Cm}-\mathrm{U}^{92+}$ collisions, respectively, as a function of the collision energy, $\eta=E / E_{0}$, where $E_{0}$ is the head-on collision energy. Assuming $K_{\mathrm{vac}}$ remains of the same order of magnitude throughout the supercritical region as at its boundary (see Fig. 22), we believe that the pair-creation probability should be only a few times less, compared to the collisions of bare nuclei. Therefore, due to relatively large values of $K_{\mathrm{vac}}$, all scenarios considered above for the collisions of bare nuclei can be applied to the collisions of bare nuclei with neutral atoms, provided the corresponding calculations of quantum dynamics of electrons are performed. In particular, the very convincing contrast in the transition from the subcritical to the supercritical $\mathrm{Cm}-\mathrm{U}$ system, which was observed for the positron spectra in collisions of bare nuclei (see Fig. 21), makes the $\mathrm{Cm}-$ $\mathrm{U}^{92+}$ collision very promising for further studies. 


\section{CONCLUSION}

We have studied possible scenarios to access QED in the supercritical Coulomb field which can be created by heavy nuclei in low-energy collisions near the Coulomb barrier. This has been done by the calculations of pair production in the collisions along the trajectories, which correspond to a given minimum internuclear distance. The clear signatures indicating the transition from the subcritical to the supercritical regime have been found in both pair-creation probabilities and positron spectra. The evidence of such a transition becomes even more convincing if one considers only a part of the positron spectrum around its maximum. For instance, it is very well pronounced in collisions of two uranium nuclei. In contrast to the previous studies by other authors, the scenarios considered do not require any sticking of colliding nuclei. Low-energy collisions of a bare uranium nucleus with neutral uranium and curium atoms have been also studied and the probability of a vacancy in the lowest energy level of a quasimolecule formed in such collisions has been evaluated. These calculations showed that the same scenario can be applied to access QED at the supercritical field in collisions of bare nuclei with neutral atoms. In particular, examinations of the changes in the positron spectra when switching from subcritical to supercritical $\mathrm{Cm}-\mathrm{U}^{92+}$ collisions are very promising for this purpose. The experimental study of the proposed scenarios, which seems feasible with the future facilities at GSI/FAIR [46-48], HIAF [49], and NICA [50], would either prove the vacuum decay in the supercritical Coulomb field or lead to discovery of a new physics, which is beyond the presently used QED formalism.

\section{ACKNOWLEDGMENTS}

We thank I. B. Khriplovich and Yu. Ts. Oganessian for stimulating discussions. This work was supported by RFBR-Rosatom (Grant No. 20-21-00098), by RFBR (Grants No. 18-03-01220, No. 20-02-00199, and No. 1832-20063), and by the President of the Russian Federation (Grant No. MK-1626.2020.2). The work of R. V. P., V. M. S., and I. A. M. was also supported by the Foundation for the advancement of theoretical physics and mathematics "BASIS". V. M. S. also acknowledges the support of the CAS President International Fellowship Initiative (PIFI) and of SPbSU (COLLAB 2019: No. 37722582). Y. S. K. acknowledges the support from the CAS PIFI under Grant No. 2018VMC0010. The work of R. V.P., N. V. K., and D. A. T. was also supported by TU Dresden via the DAAD Programm Ostpartnerschaften. A. I. B. acknowledges the support from the Ministry of Science and Higher Education of the Russian Federation (Grant No. 0784-2020-0025). The research was carried out using computational resources provided by the Resource Center "Computer Center of SPbSU."

\section{APPENDIX: PROBABILITY OF FINDING CORE HOLES}

\section{Reduced density matrix approach to a system of $N$-independent electrons}

The reduced density matrix (RDM) of $n$th order for an $N$-electron system is defined by [70,71]

$$
\begin{aligned}
\rho_{n}\left(x_{1}, \ldots, x_{n}, x_{1}^{\prime}, \ldots, x_{n}^{\prime}\right)= & \left(\begin{array}{c}
N \\
n
\end{array}\right) \int d x_{n+1}, \ldots, d x_{N} \Psi\left(x_{1}, \ldots, x_{n}, x_{n+1}, \ldots, x_{N}\right) \\
& \times \Psi^{*}\left(x_{1}^{\prime}, \ldots, x_{n}^{\prime}, x_{n+1}, \ldots, x_{N}\right),
\end{aligned}
$$

where $x$ stands for both position $(\mathbf{r})$ and bispinor $(\tau=1, \ldots, 4)$ variables, the integration over $x$ implies the integration over $\mathbf{r}$ and the summation over $\tau$, and $\Psi\left(x_{1}, \ldots, x_{N}\right)$ is the wave function of the system. We can also consider the density operator $\hat{\rho}_{n}$ as a linear integral operator with the density matrix $\rho_{n}$ being its kernel. Then, the probability $P_{n}$ of finding $n$-electron system in a state $\Phi_{n}$ is given by [71]

$$
P_{n}=n !\left\langle\Phi_{n}\left|\hat{\rho}_{n}\right| \Phi_{n}\right\rangle .
$$

Consider a system of independent $N$ electrons, described by a set of one-electron wave functions $\psi_{i}(i=1, \ldots, N)$. The $n$th order RDM in this system is given by [70,71]

$$
\rho_{n}\left(x_{1}, \ldots x_{n} ; x_{1}^{\prime}, \ldots, x_{n}^{\prime}\right)=\frac{1}{n !}\left|\begin{array}{cccc}
\rho_{1}\left(x_{1}, x_{1}^{\prime}\right) & \rho_{1}\left(x_{1}, x_{2}^{\prime}\right) & \ldots & \rho_{1}\left(x_{1}, x_{n}^{\prime}\right) \\
\rho_{1}\left(x_{2}, x_{1}^{\prime}\right) & \rho_{1}\left(x_{2}, x_{2}^{\prime}\right) & \ldots & \rho_{1}\left(x_{2}, x_{n}^{\prime}\right) \\
\ldots & \ldots & \ldots & \ldots \\
\rho_{1}\left(x_{n}, x_{1}^{\prime}\right) & \rho_{1}\left(x_{n}, x_{2}^{\prime}\right) & \ldots & \rho_{1}\left(x_{n}, x_{n}^{\prime}\right)
\end{array}\right| \text {, }
$$

where $\rho_{1}\left(x, x^{\prime}\right)$ is the one-particle density matrix defined by 


$$
\rho_{1}\left(x, x^{\prime}\right)=\sum_{i=1}^{N} \psi_{i}(x) \psi_{i}^{*}\left(x^{\prime}\right)
$$

The wave function $\Phi_{n}$ of an $n$-electron state is given by the Slater determinant constructed from one-electron functions $\varphi_{k}$. In this case it is easy to show that the probability $P_{n}$ defined by Eq. (A2) is equal to the determinant of an $n \times n$ matrix $\gamma$ constructed from the one-electron density matrix,

$$
P_{n}=\operatorname{det} \gamma, \quad \gamma_{k j}=\left\langle\varphi_{k}\left|\hat{\rho}_{1}\right| \varphi_{j}\right\rangle,
$$

where $\hat{\rho}_{1}$ is the one-particle density operator defined by

$$
\hat{\rho}_{1}=\sum_{i \in \text { occupied }}\left|\psi_{i}\right\rangle\left\langle\psi_{i}\right|
$$

Thus, $P_{n}$ is the probability of finding $n$ states occupied in the $N$-electron system while the remaining $N-n$ electrons are not detected. Equation (A5) coincides with that obtained by the method of inclusive probabilities (see Refs. $[68,69]$ and references therein).

We can also introduce the probability $\bar{P}_{m}$ of finding $m$ holes in an $N$-electron system by defining the one-hole density matrix $\bar{\rho}_{1}$. Due to completeness of the set of functions $\psi_{i}(x)$ the hole density operator can be written in the form

$$
\hat{\bar{\rho}}_{1}=\sum_{i \in \text { vacant }}\left|\psi_{i}\right\rangle\left\langle\psi_{i}\right|=\hat{I}-\hat{\rho}_{1} .
$$

Then, we get

$$
\bar{P}_{m}=\operatorname{det} \bar{\gamma}, \quad \bar{\gamma}_{k j}=\left\langle\varphi_{k}\left|\hat{\bar{\rho}}_{1}\right| \varphi_{j}\right\rangle=\delta_{k j}-\gamma_{k j} .
$$

\section{Probability of finding one or two core holes $(m=2)$}

Consider an $N$-electron system and two one-electron wave functions $\varphi_{1}(x)$ and $\varphi_{2}(x)$ which correspond to $1 \sigma$ states with the angular momentum projection $\mu=1 / 2$ and $\mu=-1 / 2$, respectively. We denote by $A$ the event when $1 \sigma_{1 / 2}$ is occupied by electron and by $\bar{A}$ the event when this state is vacant. Similarly, the event $B$ means that the state $1 \sigma_{-1 / 2}$ is occupied and the event $\bar{B}$ means that $1 \sigma_{-1 / 2}$ is vacant.

Thus, four incompatible events can occur: $A B, A \bar{B}, \bar{A} B$ and $\bar{A} \bar{B}$, which correspond to the cases: both states are occupied, the first state is occupied and the second state is vacant, the first state is vacant and the second state is occupied, and both states are vacant. Using Eqs. (A5) and (A8), we obtain for the corresponding probabilities

$$
\begin{aligned}
& P(A B)=\gamma_{11} \gamma_{22}-\left|\gamma_{12}\right|^{2}, \\
& P(\bar{A} \bar{B})=\left(1-\gamma_{11}\right)\left(1-\gamma_{22}\right)-\left|\gamma_{12}\right|^{2} .
\end{aligned}
$$

Using $P(\bar{A} B)=P(\bar{A})-P(\bar{A} \bar{B})$, we obtain

$$
\begin{aligned}
& P(A \bar{B})=\gamma_{11}\left(1-\gamma_{22}\right)+\left|\gamma_{12}\right|^{2}, \\
& P(\bar{A} B)=\left(1-\gamma_{11}\right) \gamma_{22}+\left|\gamma_{12}\right|^{2} .
\end{aligned}
$$

With the help of the total probability rule, for the probability of pair creation with the occupation of at least one $1 \sigma$ vacancy, $P(C)$, we obtain

$$
\begin{aligned}
P(C)= & P(C / A B) \cdot P(A B)+P(C / \bar{A} B) \cdot P(\bar{A} B) \\
& +P(C / A \bar{B}) \cdot P(A \bar{B})+P(C / \bar{A} \bar{B}) \cdot P(\bar{A} \bar{B}) .
\end{aligned}
$$

Here $P(C / D)$ is the conditional probability, i.e., the probability of occurring the event $C$, given the event $D$ has occurred. Assuming the pair-creation probability is small enough, we obtain

$$
P(C / \bar{A} \bar{B}) \simeq P(C / \bar{A} B)+P(C / A \bar{B}) .
$$

Then, taking into account that $P(C / A B)=0$, we get

$$
P(C) \simeq P(C / \bar{A} B)\left[1-\gamma_{11}\right]+P(C / A \bar{B})\left[1-\gamma_{22}\right] .
$$

Assuming the states with $\mu= \pm 1 / 2$ are equivalent, which means

$$
\gamma_{11}=\gamma_{22}, \quad P(C / \bar{A} B)=P(C / A \bar{B}),
$$

we have

$$
P(C) \simeq 2 P(C / \bar{A} B) K_{\mathrm{vac}},
$$

where

$$
K_{\mathrm{vac}}=P(\bar{A})=1-\gamma_{11} .
$$


[1] F. Sauter, Z. Phys. 69, 742 (1931).

[2] W. Heisenberg and H. Euler, Z. Phys. 98, 714 (1936).

[3] J. Schwinger, Phys. Rev. 82, 664 (1951).

[4] E. S. Fradkin, D. M. Gitman, and S. M. Shvartsman, Quantum Electrodynamics with Unstable Vacuum (SpringerVerlag, Berlin, 1991).

[5] A. Di Piazza, C. Müller, K. Z. Hatsagortsyan, and C. H. Keitel, Rev. Mod. Phys. 84, 1177 (2012).

[6] S. P. Gavrilov and D. M. Gitman, Phys. Rev. D 53, 7162 (1996).

[7] H. K. Avetissian, A. K. Avetissian, G. F. Mkrtchian, and Kh. V. Sedrakian, Phys. Rev. E 66, 016502 (2002).

[8] N. B. Narozhny, S. S. Bulanov, V. D. Mur, and V. S. Popov, Phys. Lett. A 330, 1 (2004); JETP Lett. 80, 382 (2004); S. S. Bulanov, V. D. Mur, N. B. Narozhny, J. Nees, and V. S. Popov, Phys. Rev. Lett. 104, 220404 (2010).

[9] R. Schützhold, H. Gies, and G. Dunne, Phys. Rev. Lett. 101, 130404 (2008).

[10] M. Ruf, G. R. Mocken, C. Müller, K. Z. Hatsagortsyan, and C. H. Keitel, Phys. Rev. Lett. 102, 080402 (2009).

[11] N. Abdukerim, Z. Li, and B. Xie, Phys. Lett. B 726, 820 (2013); Chin. Phys. B 26, 020301 (2017).

[12] A. Otto, D. Seipt, D. Blaschke, B. Kämpfer, and S. A. Smolyansky, Phys. Lett. B 740, 335 (2015); A. Otto, D. Seipt, D. Blaschke, S. A. Smolyansky, and B. Kämpfer, Phys. Rev. D 91, 105018 (2015).

[13] S. P. Gavrilov and D. M. Gitman, Phys. Rev. D 95, 076013 (2017).

[14] G. Torgrimsson, C. Schneider, and R. Schützhold, Phys. Rev. D 97, 096004 (2018).

[15] C. Kohlfürst and R. Alkofer, Phys. Rev. D 97, 036026 (2018).

[16] I. A. Aleksandrov, G. Plunien, and V. M. Shabaev, Phys. Rev. D 97, 116001 (2018); 99, 016020 (2019).

[17] A. Ringwald, Phys. Lett. B 510, 107 (2001).

[18] G. A. Mourou, T. Tajima, and S. V. Bulanov, Rev. Mod. Phys. 78, 309 (2006).

[19] I. Pomeranchuk and J. Smorodinsky, J. Phys. USSR 9, 97 (1945).

[20] S. S. Gershtein and Y. B. Zeldovich, Zh. Eksp. Teor. Fiz. 57, 654 (1969); [Sov. Phys. JETP 30, 358 (1970)]; Lett. Nuovo Cimento 1, 835 (1969).

[21] W. Pieper and W. Greiner, Z. Phys. 218, 327 (1969).

[22] V. S. Popov, Pis'ma Zh. Eksp. Teor. Fiz. 11, 254 (1970) [JETP Lett. 11, 162 (1970)]; Yad. Fiz. 12, 429 (1970) [Sov. J. Nucl. Phys. 12, 235 (1971)]; Zh. Eksp. Teor. Fiz. 59, 965 (1970) [Sov. Phys. JETP 32, 526 (1971)]; Zh. Eksp. Teor. Fiz. 60, 1228 (1971) [Sov. Phys. JETP 33, 665 (1971)].

[23] Y. B. Zeldovich and V. S. Popov, Usp. Fiz. Nauk 105, 403 (1971) [Sov. Phys. Usp. 14, 673 (1972)].

[24] B. Müller, H. Peitz, J. Rafelski, and W. Greiner, Phys. Rev. Lett. 28, 1235 (1972); B. Müller, J. Rafelski, and W. Greiner, Z. Phys. 257, 62 (1972); 257, 183 (1972).

[25] V. D. Mur and V. S. Popov, Teor. Mat. Fiz. 27, 204 (1976) [Theor. Math. Phys. 27, 429 (1976)].

[26] V. S. Popov, V. L. Eletsky, and V. D. Mur, Zh. Eksp. Teor. Fiz. 71, 856 (1976) [Sov. Phys. JETP 44, 451 (1976)].

[27] B. Müller, Annu. Rev. Nucl. Sci. 26, 351 (1976).

[28] J. Reinhardt and W. Greiner, Rep. Prog. Phys. 40, 219 (1977).
[29] G. Soff, J. Reinhardt, B. Müller, and W. Greiner, Phys. Rev. Lett. 38, 592 (1977).

[30] J. Rafelski, L. P. Fulcher, and A. Klein, Phys. Rep. 38, 227 (1978).

[31] J. Reinhardt, B. Müller, and W. Greiner, Phys. Rev. A 24, 103 (1981).

[32] W. Greiner, B. Müller, and J. Rafelski, Quantum Electrodynamics of Strong Fields (Springer-Verlag, Berlin, 1985).

[33] U. Müller, T. de Reus, J. Reinhardt, B. Müller, W. Greiner, and G. Soff, Phys. Rev. A 37, 1449 (1988).

[34] U. Müller-Nehler and G. Soff, Phys. Rep. 246, 101 (1994).

[35] J. Reinhardt and W. Greiner, Supercritical fields and the decay of the vacuum, in Proceeding of the Memorial Symposium for Gerhard Soff, edited by W. Greiner and J. Reinhardt (EP Systema, Budapest, 2005), pp. 181-192.

[36] J. Rafelski, J. Kirsch, B. Müller, J. Reinhardt, and W. Greiner, in New Horizons in Fundamental Physics, edited by S. Schramm and M. Schafer, FIAS Interdisciplinary Science Series (Springer, 2016), pp. 211-251, https://doi .org/10.1007/978-3-319-44165-8.

[37] I. I. Tupitsyn, Y. S. Kozhedub, V. M. Shabaev, G. B. Deyneka, S. Hagmann, C. Kozhuharov, G. Plunien, and Th. Stöhlker, Phys. Rev. A 82, 042701 (2010).

[38] I. I. Tupitsyn, Y. S. Kozhedub, V. M. Shabaev, A. I. Bondarev, G. B. Deyneka, I. A. Maltsev, S. Hagmann, G. Plunien, and Th. Stöhlker, Phys. Rev. A 85, 032712 (2012).

[39] G. B. Deyneka, I. A. Maltsev, I. I. Tupitsyn, V. M. Shabaev, and G. Plunien, Russ. J. Phys. Chem. B 6, 224 (2012).

[40] G. B. Deyneka, I. A. Maltsev, I. I. Tupitsyn, V. M. Shabaev, A. I. Bondarev, Y.S. Kozhedub, G. Plunien, and Th. Stöhlker, Eur. Phys. J. D 67, 258 (2013).

[41] Y.S. Kozhedub, V. M. Shabaev, I. I. Tupitsyn, A. Gumberidze, S. Hagmann, G. Plunien, and Th. Stöhlker, Phys. Rev. A 90, 042709 (2014).

[42] I. A. Maltsev, V. M. Shabaev, I. I. Tupitsyn, Y. S. Kozhedub, G. Plunien, and Th. Stöhlker, Nucl. Instrum. Methods Phys. Res., Sect. B 408, 97 (2017).

[43] R. V. Popov, A. I. Bondarev, Y. S. Kozhedub, I. A. Maltsev, V. M. Shabaev, I. I. Tupitsyn, X. Ma, G. Plunien, and Th. Stöhlker, Eur. Phys. J. D 72, 115 (2018).

[44] I. A. Maltsev, V. M. Shabaev, R. V. Popov, Y. S. Kozhedub, G. Plunien, X. Ma, and Th. Stöhlker, Phys. Rev. A 98, 062709 (2018).

[45] I. A. Maltsev, V. M. Shabaev, R. V. Popov, Y. S. Kozhedub, G. Plunien, X. Ma, and Th. Stöhlker, and D. A. Tumakov, Phys. Rev. Lett. 123, 113401 (2019).

[46] A. Gumberidze, Th. Stöhlker, H. F. Beyer, F. Bosch, A. Bräuning-Demian, S. Hagmann, C. Kozhuharov, Th. Kühl, R. Mann, P. Indelicato, W. Quint, R. Schuch, and A. Warczak, Nucl. Instrum. Methods Phys. Res., Sect. B 267, 248 (2009).

[47] M. Lestinsky et al., Eur. Phys. J. Special Topics 225, 797 (2016).

[48] S. Hagmann, P.-M. Hillenbrand, Yu. Litvinov, U. Spillmann, V. Shabaev, I. Tupitsyn, E. de Filippo, M. Schoeffler, L. Schmidt, Ch. Kozhuharov, M. Benis, A. Gumberidze, M. Lestinski, N. Petridis, H. Rothard, and Th. Stöhlker, Proposal for an experiment to be conducted at ESR (to be published). 
[49] X. Ma, W. Q. Wen, S. F. Zhang, D. Y. Yu, R. Cheng, J. Yang, Z. K. Huang, H. B. Wang, X. L. Zhu, X. Cai, Y. T. Zhao, L. J. Mao, J. C. Yang, X. H. Zhou, H. S. Xu, Y. J. Yuan, J. W. Xia, H. W. Zhao, G. Q. Xiao, and W. L. Zhan, Nucl. Instrum. Methods Phys. Res., Sect. B 408, 169 (2017).

[50] G. M. Ter-Akopian, W. Greiner, I. N. Meshkov, Y. T. Oganessian, J. Reinhardt, and G. V. Trubnikov, Int. J. Mod. Phys. E 24, 1550016 (2015).

[51] I. A. Maltsev, V. M. Shabaev, I. I. Tupitsyn, A. I. Bondarev, Y. S. Kozhedub, G. Plunien, and Th. Stöhlker, Phys. Rev. A 91, 032708 (2015).

[52] A. I. Bondarev, I. I. Tupitsyn, I. A. Maltsev, Y. S. Kozhedub, and G. Plunien, Eur. Phys. J. D 69, 110 (2015).

[53] J. Sapirstein and W. R. Johnson, J. Phys. B 29, 5213 (1996).

[54] V. M. Shabaev, I. I. Tupitsyn, V. A. Yerokhin, G. Plunien, and G. Soff, Phys. Rev. Lett. 93, 130405 (2004).

[55] J. Crank and P. Nicolson, Proc. Cambridge Philos. Soc. 43, 50 (1947).

[56] P. W. Langhoff, J. Sims, and C. T. Corcoran, Phys. Rev. A 10, 829 (1974).

[57] L. D. Landau and E. M. Lifschitz, Mechanics, 3rd ed. (Butterworth- Heinemann, Oxford, 1982).

[58] H. Backe, L. Handschug, F. Hessberger, E. Kankeleit, L. Richter, F. Weik, R. Willwater, H. Bokemeyer, P. Vincent, Y. Nakayama, and J. S. Greenberg, Phys. Rev. Lett. 40, 1443 (1978).

[59] C. Kozhuharov, P. Kienle, E. Berdermann, H. Bokemeyer, J. S. Greenberg, Y. Nakayama, P. Vincent, H. Backe,
L. Handschug, and E. Kankeleit, Phys. Rev. Lett. 42, 376 (1979).

[60] N. Moiseyev, Phys. Rep. 302, 212 (1998).

[61] E. Lindroth and L. Argenti, Adv. Quantum Chem. 63, 247 (2012).

[62] E. Ackad and M. Horbatsch, Phys. Rev. A 75, 022508 (2007); 76, 022503 (2007).

[63] A. Marsman and M. Horbatsch, Phys. Rev. A 84, 032517 (2011).

[64] V. A. Zaytsev, I. A. Maltsev, I. I. Tupitsyn, and V. M. Shabaev, Phys. Rev. A 100, 052504 (2019); V. A. Zaytsev, I. A. Maltsev, I. I. Tupitsyn, V. M. Shabaev, and V. Yu. Ivanov, Opt. Spectrosc. 128, 307 (2020).

[65] S. I. Godunov, B. Machet, and M. I. Vysotsky, Eur. Phys. J. C 77, 782 (2017).

[66] I. A. Maltsev, V. M. Shabaev, V. A. Zaytsev, R. V. Popov, Y. S. Kozhedub, and D. A. Tumakov, Opt. Spectrosc. 128, 1100 (2020).

[67] J. P. Perdew and A. Zunger, Phys. Rev. B 23, 5048 (1981).

[68] H. J. Lüdde and R. M. Dreizler, J. Phys. B 18, 107 (1985).

[69] T. Kirchner, H. J. Lüdde, and M. Horbatsch, Recent Res. Dev. Phys. Chem. 5, 433 (2004).

[70] R. McWeeny, Methods of Molecular Quantum Mechanics (Academic Press, London, 1992).

[71] E. R. Davidson, Reduced Density Matrices in Quantum Chemistry, Theoretical Chemistry, Vol. 6 (Academic Press, New York, 1976). 